NISSUNA UMANA INVESTIGAZIONE SI PUO DIMANDARE VERA SCIENZIA S'ESSA NON PASSA PER LE MATEMATICHE DIMOSTRAZIONI LEONARDO DA VINCI

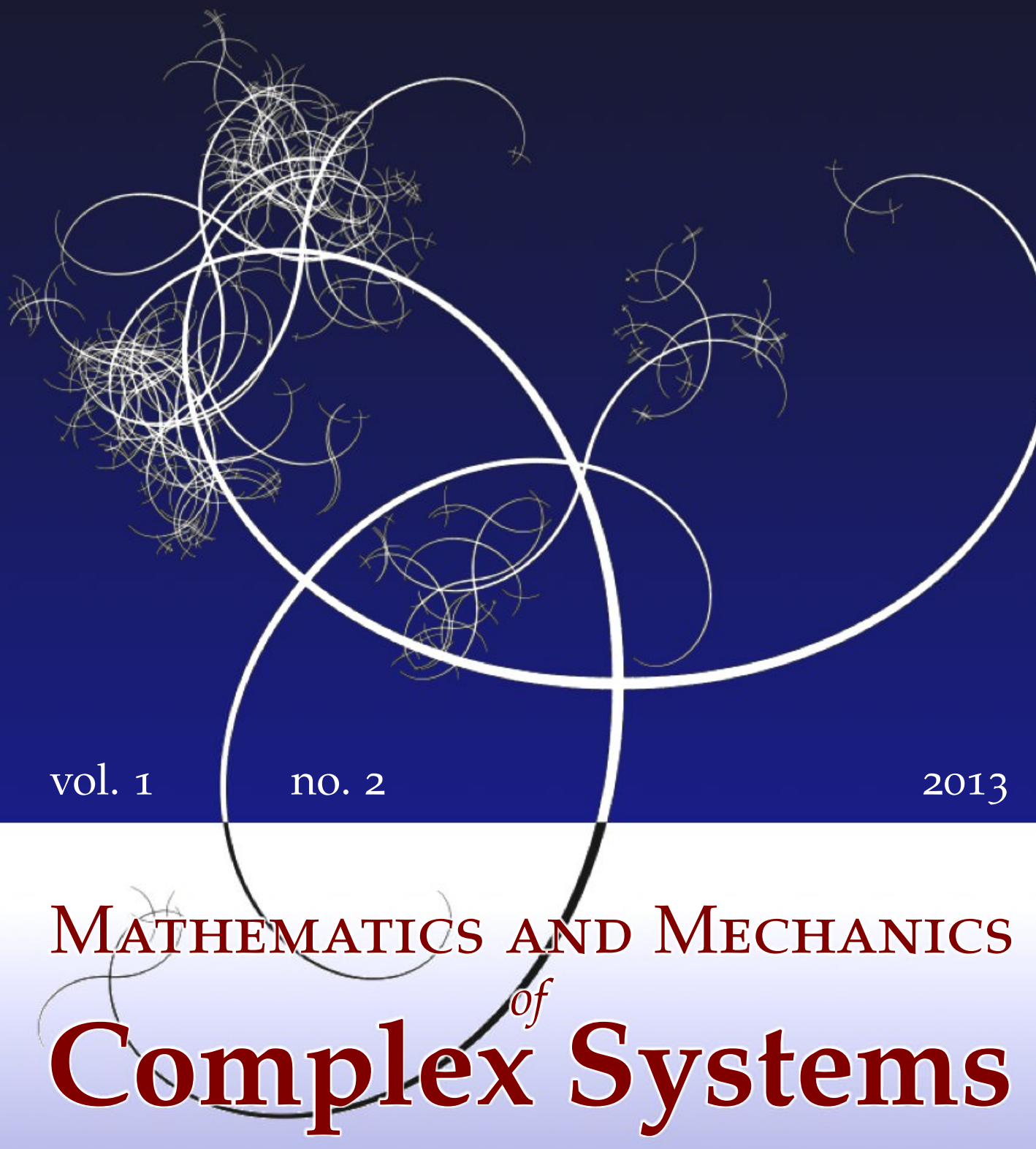

Marc Olive And Nicolas AufFray

SYMMETRY CLASSES FOR EVEN-ORDER TENSORS 


\title{
SYMMETRY CLASSES FOR EVEN-ORDER TENSORS
}

\author{
Marc Olive And Nicolas AufFray
}

\begin{abstract}
We give a complete general answer to the problem, recurrent in continuum mechanics, of determining of the number and type of symmetry classes of an even-order tensor space. This kind of investigation was initiated for the space of elasticity tensors, and since then different authors have solved this problem for other kinds of physics, such as photoelectricity, piezoelectricity, flexoelectricity, and strain-gradient elasticity. All these problems were treated using the same computational method, which, though effective, has the drawback of not providing general results. Furthermore, its complexity increases with the tensorial order. Here we provide general theorems that directly give the desired results for any even-order constitutive tensor. As an illustration of this method, and for the first time, the symmetry classes of all even-order tensors of Mindlin second strain-gradient elasticity are provided.
\end{abstract}

\section{Introduction}

Physical motivation. In the last years there has been increased interest in generalized continuum theories; see, for example, [Forest 1998; dell'Isola et al. 2009; 2012; Lebée and Sab 2011]. These works, based on the pioneering articles [Toupin 1962; Mindlin 1964; 1965], propose extended kinematic formulations, to take into account size effects within the continuum. The price to be paid for this is the appearance of tensors of order greater than four in the constitutive relations. These higher-order objects are difficult to handle and extracting physically meaningful information from them is not straightforward. The aim of this paper is to provide general results concerning the type and number of anisotropic systems an evenorder tensor can have.

Such results have important applications, at least, for the modeling and numerical implementation of nonclassical linear constitutive laws:

Modeling: The purpose of modeling is, given a material and a set of physical variables of interest, to construct the more general constitutive law (a linear one, in the present context) that describes the behavior of that material. An example of such a method is provided in [Thionnet and Martin 2006], where,

MSC2010: 15A72, 20C35, 74B99.

Keywords: anisotropy, symmetry classes, higher-order tensors, generalized continuum theories, strain-gradient elasticity. 
given a set of variables $V$ and the material symmetry group $S$, the authors derive mechanical behavior laws using the theory of invariants and continuum thermodynamics. In such regard our results will say, without making any computation whether or not $S$ is contained in the set of symmetry classes of $\mathscr{L}\left(v, v^{\prime}\right)$ the space of linear maps from $v \in V$ to $v^{\prime} \in V$.

Numerical implementation: To implement a new linear constitutive law in a finite-element code, one has to know the complete set of matrices needed to model the associated anisotropic behavior. In that regard, our result is a precious guideline, as it tells you how many matrices there are and how to construct them. This is illustrated in the case of three-dimensional strain gradient elasticity in [Auffray et al. 2013].

Constitutive tensors symmetry classes. In mechanics, constitutive laws are usually expressed in terms of tensorial relations between the gradients of primary variables and their fluxes [Gu and He 2011]. As is well known, this feature is not restricted to linear behaviors, since tensorial relations appear in the tangential formulation of nonlinear ones [Triantafyllidis and Bardenhagen 1996]. It is also known that a general tensorial relation can be divided into classes according to its symmetry properties. Such classes are known in mechanics as symmetry classes [Forte and Vianello 1996], and in mathematical physics as isotropic classes or strata [Abud and Sartori 1983; Auffray et al. 2011].

In the case of second-order tensors, the determination of symmetry classes is rather simple. Using spectral analysis it can be concluded that any second-order symmetric tensor ${ }^{1}$ can either be orthotropic $\left(\left[\mathrm{D}_{2}\right]\right)$, transverse isotropic $([\mathrm{O}(2)])$, or isotropic ([SO(3)]). Such tensors are commonly used to describe, for example, heat conduction and electric permittivity.

For higher-order tensors, the determination of the set of symmetry classes is more involved, and is mostly based on an approach introduced in [Forte and Vianello 1996] for the case of elasticity. Let us briefly detail this case.

The vector space of elasticity tensors, denoted by $\mathbb{E}$ la throughout this paper, is the subspace of fourth-order tensors endowed with the following index symmetries:

Minor symmetries: $E_{i j k l}=E_{j i k l}=E_{j i l k}$.

Major symmetry: $E_{i j k l}=E_{k l i j}$.

Symmetries will be specified using notation such as $E_{(i j)(k l)}$, where (..) indicates invariance under permutation of the indices in parentheses, and $\ldots \ldots$ indicates invariance with respect to permutations of the underlined blocks. Index symmetries encode the physics described by the mathematical operator. The minor symmetries

\footnotetext{
${ }^{1}$ Such a tensor is related to a symmetric matrix, which can be diagonalized in an orthogonal basis. The stated result is related to this diagonalization.
} 
stem from the fact that rigid body motions do not induce deformation (the symmetry of $\varepsilon$ ), and that the material is not subjected to volumic couple (the symmetry of $\sigma$ ). The major symmetry is the consequence of the existence of free energy. An elasticity tensor, $\mathbf{E}$, can be viewed as a symmetric linear operator on $\mathbb{T}_{(i j)}$, the space of symmetric second-order tensors. According to [Forte and Vianello 1996], for the classical action of $\mathrm{SO}(3)$, Ela is divided into eight symmetry classes (see page 183 for the notation):

$$
[\mathbb{E l a}]=\left\{[\mathbb{1}],\left[\mathrm{Z}_{2}\right],\left[\mathrm{D}_{2}\right],\left[\mathrm{D}_{3}\right],\left[\mathrm{D}_{4}\right],[\mathrm{O}(2)],[\mathrm{O}],[\mathrm{SO}(3)]\right\},
$$

which correspond, respectively, to the following physical classes: ${ }^{2}$ triclinic, monoclinic, orthotropic, trigonal, tetragonal, transverse isotropic, cubic, and isotropic. Besides this fundamental result, the interest of the Forte and Vianello paper was to provide a general method to determine the symmetry classes of any tensor space [Auffray et al. 2011]. Other results have been obtained by this method since then:

\begin{tabular}{|clcrl|}
\hline Property & Tensor & $\begin{array}{c}\text { Number } \\
\text { of classes }\end{array}$ & Action & Studied in \\
Photoelasticity & $T_{(i j)(k l)}$ & 12 & $\mathrm{SO}(3)$ & [Forte and Vianello 1997] \\
Piezoelectricity & $T_{(i j) k}$ & 15 & $\mathrm{O}(3)$ & [Geymonat and Weller 2002] \\
$\begin{array}{c}\text { Flexoelectricity } \\
\begin{array}{c}\text { A set of tensors } \\
\text { of order six }\end{array}\end{array} T_{(i j) k l}$ & 12 & $\mathrm{SO}(3)$ & [Le Quang and He 2011] \\
\hline
\end{tabular}

The limitations of the Forte-Vianello approach. The method introduced by Forte and Vianello is actually the most general. ${ }^{3}$ But, at the same time, it suffers from at least two limitations:

(1) The computation of the harmonic decomposition.

(2) The specificity of the study for each kind of tensor.

In its original setting, the method requires the computation of the explicit harmonic decomposition of the studied tensor, that is, its decomposition into the sum of its $\mathrm{SO}(3)$-irreducible components, also known as harmonic tensors ${ }^{4}$. Its explicit computation, which is generally based on an algorithm introduced by Spencer [1970], turns out to be intractable in practice as the tensorial order increases. But

\footnotetext{
${ }^{2}$ These symmetry classes are subgroups of the group $\mathrm{SO}(3)$ of space rotations. This is because the elasticity tensor is of even order. To treat odd-order tensors, the full orthogonal group $\mathrm{O}(3)$ has to be considered.

${ }^{3}$ Some other methods can be found in the literature, such as counting the symmetry planes [Chadwick et al. 2001], or studying the SU(2)-action on Ela [Bóna et al. 2004], and others, but these methods are difficult to generalize to arbitrary vector spaces.

${ }^{4}$ Harmonic tensors are completely symmetric and traceless. They inherit this name because of a well-known isomorphism in $\mathbb{R}^{3}$ between these tensors and harmonic polynomials [Backus 1970].
} 
this is not a real problem, since the only information needed is the number of different harmonic tensors of each order appearing in the decomposition, that is, the isotypic decomposition. Based on arguments presented in [Jerphagnon et al. 1978], there exists a direct procedure to obtain this isotypic decomposition from the tensor index symmetries [Auffray 2008]. Such an approach has been used in [Le Quang et al. 2012] to obtain the symmetry classes of sixth-order tensors.

As each kind of tensor space requires specific study, this specificity constitutes the other limitation of the method. This remark has to be considered together with the observation that, for even-order tensors it seems that there exist only two possibilities. Namely, a tensor space has as many classes as

- the full symmetric tensor space (for example, Ela is divided into eight classes, like the full symmetric tensor space [Forte and Vianello 1996]), or

- the generic tensor space ${ }^{5}$ (other fourth-order tensor spaces such as those of photoelasticity [Forte and Vianello 1997], flexoelectricity [Le Quang and $\mathrm{He}$ 2011], etc., are divided into 12 classes, like the generic tensor space).

The same observation can also be made for second and sixth-order tensors [Le Quang et al. 2012]. Understanding the general rule behind this observation would be an important result in mechanics. Its practical implication is the direct determination of the number and the type of symmetry classes for any constitutive law, no matter its order. This result is valuable for understanding generalized continuum theories, in which higher-order tensors are involved in constitutive laws.

Organization of the paper. In Section 2, the main results of this paper, Theorems I, II, and III, are stated. As an application, the symmetry classes of the even-order constitutive tensor spaces of Mindlin second strain-gradient elasticity are determined. Results concerning the sixth-order coupling tensor and the eighth-order second strain-gradient tensor are given for the first time. Obtaining the same results with the Forte-Vianello approach would have been much more difficult. Other sections are dedicated to the construction of our proofs. In Section 3, the mathematical framework used to obtain our result is introduced. Thereafter, we study the symmetry classes of a couple of harmonic tensors, which is the main purpose of the tool named the clips operator. We then give the associated results for couples of SO(3)-closed subgroups (Theorem 4.6 and Table 2). Thanks to these results, and with the help of previous work on the topic [Ihrig and Golubitsky 1984], we obtain in Section 5 some general results concerning symmetry classes for general evenorder tensors. In Section 6 our main results are finally proved. The Appendix is devoted to proofs and the calculus of clips operations.

${ }^{5}$ The $n$-th order generic tensor is a $n$-th order tensor with no index symmetry. 


\section{Main results}

In this section, our main results are stated. In the first subsection, the construction of constitutive tensor spaces (CTS) is discussed. This construction allows us to formulate our main results in the next subsection. Finally, the application of these results to Mindlin second strain-gradient elasticity (SSGE) is considered. Precise mathematical definitions of the symmetry classes are given in Section 3.

Construction of CTS. Linear constitutive laws are linear maps between the gradients of primary physical quantities and their fluxes. Each of these physical quantities (see Table 1 on the next page) is in fact related to subspaces ${ }^{6}$ of tensors spaces; these subspaces will be called state tensor spaces (STS). These STS will be the primitive notion from which the CTS will be constructed.

Notation. $\mathscr{L}(F, G)$ will indicate the vector space of linear maps from $F$ to $G$.

Now we consider two STS, $\mathbb{E}_{1}=\mathbb{T}_{G}$ and $\mathbb{E}_{2}=\mathbb{T}_{f}$, respectively of order $p$ and order $q$, possibly with index symmetries. As a consequence, they belong to subspaces of $\bigotimes^{p} \mathbb{R}^{3}$ and $\bigotimes^{q} \mathbb{R}^{3}$. A constitutive tensor $C$ is a linear map between $\mathbb{E}_{1}$ and $\mathbb{E}_{2}$, that is, an element of the space $\mathscr{L}\left(\mathbb{E}_{1}, \mathbb{E}_{2}\right)$. This space is isomorphic, modulo the use of an euclidean metric, to $\mathbb{E}_{1} \otimes \mathbb{E}_{2}$. Physical properties lead to some index symmetries on $C \in \mathbb{E}_{1} \otimes \mathbb{E}_{2}$; thus the vector space of such $C$ is some vector subspace $\mathbb{T}_{C}$ of $\mathbb{E}_{1} \otimes \mathbb{E}_{2}$.

Now, each of the spaces $\mathbb{E}_{1}, \mathbb{E}_{2}$, and $\mathbb{E}_{1} \otimes \mathbb{E}_{2}$ has natural $\mathrm{O}(3)$ actions. In this paper, we are concerned with cases in which $p+q=2 n$. In such a situation, it is known that the $\mathrm{O}(3)$-action on $\mathbb{E}_{1} \otimes \mathbb{E}_{2}$ reduces to that of $\mathrm{SO}(3)$ [Forte and Vianello 1996]. We therefore have

$$
\mathscr{L}\left(\mathbb{E}_{1}, \mathbb{E}_{2}\right) \simeq \mathbb{E}_{1} \otimes \mathbb{E}_{2} \subset \mathbb{T}^{p} \otimes \mathbb{T}^{q}=\mathbb{T}^{p+q=2 n} .
$$

Here are some examples of this construction:

\begin{tabular}{|c|c|c|c|c|}
\hline Property & $\mathbb{E}_{1}$ & $\mathbb{E}_{2}$ & $\begin{array}{l}\text { Tensor product } \\
\text { for CTS }\end{array}$ & $\begin{array}{l}\text { Number } \\
\text { of classes }\end{array}$ \\
\hline Elasticity & $\mathbb{T}_{(i j)}$ & $\mathbb{T}_{(i j)}$ & Symmetric & 8 \\
\hline Photoelasticity & $\mathbb{T}_{(i j)}$ & $\mathbb{T}_{(i j)}$ & Standard & 12 \\
\hline Flexoelectricity & $\mathbb{T}_{(i j) k}$ & $\mathbb{T}_{i}$ & Standard & 12 \\
\hline First-gradient elasticity & $\mathbb{T}_{(i j) k}$ & $\mathbb{T}_{(i j) k}$ & Symmetric & 17 \\
\hline
\end{tabular}

This table shows two kinds of CTS, describing respectively:

- coupled physics (tensors such as photoelasticity and flexoelectricity, encoding the coupling between two different physics), and

\footnotetext{
${ }^{6}$ Because of some symmetries.
} 


\begin{tabular}{|lll|}
\hline Physical notion & Mathematical object & Mathematical space \\
Gradient & Tensor state $\mathbf{T}_{1} \in \bigotimes^{p} \mathbb{R}^{3}$ & $\mathbb{\mathbb { T }}_{G}$ : tensor space with \\
& index symmetries \\
Fluxes of gradient & Tensor state $\mathbf{T}_{2} \in \bigotimes^{q} \mathbb{R}^{3}$ & $\mathbb{T}_{f}:$ tensor space with \\
& & index symmetries \\
Linear constitutive law & $C \in \mathscr{L}\left(\mathbb{T}_{G}, \mathbb{T}_{f}\right)$ & $\mathbb{T}_{C} \subset \mathscr{L}\left(\mathbb{T}_{G}, \mathbb{T}_{f}\right)$ \\
\hline
\end{tabular}

Table 1. Physical and mathematical links.

- proper physics (tensors such as classical and first-gradient elasticities, describing a single physical phenomenon).

On the mathematical side this implies:

- Coupled physics: the spaces $\mathbb{E}_{1}$ and $\mathbb{E}_{2}$ may differ, and when $\mathbb{E}_{1}=\mathbb{E}_{2}$ linear maps are not self-adjoint.

- Proper physics: we have $\mathbb{E}_{1}=\mathbb{E}_{2}$ and linear maps are self-adjoint. ${ }^{7}$

Therefore, the elasticity tensor is a self-adjoint linear map between the vector space of deformation tensors and the vector space of stress tensors. These two spaces are modeled on $\mathbb{T}_{(i j)}$. The vector space of elasticity tensors is therefore completely determined by $\mathbb{T}_{(i j)}$ and the symmetric nature of the tensor product, that is, $\mathbb{E l a}=\mathbb{T}_{(i j)} \otimes{ }^{S} \mathbb{T}_{(k l)}$, where $\otimes^{S}$ denotes the symmetric tensor product. On the side of coupling tensors, flexoelectricity is a linear map between $\mathbb{E}_{1}=\mathbb{T}_{(i j) k}$, the space of deformation gradients, and $\mathbb{E}_{2}=\mathbb{\mathbb { V }}_{l}$, the electric polarization; therefore $\mathbb{F l e x}=\mathbb{T}_{(i j) k} \otimes \mathbb{T}_{l}$.

Symmetry classes of even-order tensor spaces. Consider an even-order CTS $\mathbb{T}^{2 n}$. It is known [Jerphagnon et al. 1978] that this space can be decomposed orthogonally ${ }^{8}$ into a full symmetric space and a complementary one which is isomorphic to a tensor space of order $2 n-1$, that is:

$$
\mathbb{T}^{2 n}=\mathbb{S}^{2 n} \oplus \mathbb{C}^{2 n-1} \text {. }
$$

Let us introduce:

$\mathbb{S}^{2 n}:$ the vector space of $2 n$-th order completely symmetric tensors.

$\mathbb{G}^{2 n}$ : the vector space of $2 n$-th order tensors with no index symmetries. ${ }^{9}$

The following observation is obvious:

$$
\mathbb{S}^{2 n} \subseteq \mathbb{T}^{2 n} \subseteq \mathbb{G}^{2 n},
$$

\footnotetext{
${ }^{7}$ This is a consequence of the assumption of the existence of a free energy.

${ }^{8}$ The related dot product is constructed by $2 n$ products of the $\mathbb{R}^{3}$ canonical one.

${ }^{9}$ Formally this space is constructed as $\mathbb{G}^{2 n}=\bigotimes^{2 n} \mathbb{R}^{3}$.
} 
and therefore, if we denote by $\mathfrak{I}$ the operator which gives to a tensor space the set of its symmetry classes, we obtain:

$$
\Im\left(\mathbb{S}^{2 n}\right) \subseteq \Im\left(\mathbb{T}^{2 n}\right) \subseteq \mathfrak{I}\left(\mathbb{G}^{2 n}\right) .
$$

The symmetry group of even-order tensors is conjugate to $\mathrm{SO}(3)$-closed subgroups [Zheng and Boehler 1994; Forte and Vianello 1996]. Classification of SO(3)-closed subgroups is a classical result that can be found in many references [Ihrig and Golubitsky 1984; Sternberg 1994]. These subgroups are, up to conjugacy:

Lemma 2.1. Every closed subgroup of $\mathrm{SO}(3)$ is conjugate to precisely one group from the following list:

$$
\left\{\mathbb{1}, \mathrm{Z}_{n}, \mathrm{D}_{n}, \mathscr{T}, \mathcal{O}, \mathscr{T}, \mathrm{SO}(2), \mathrm{O}(2), \mathrm{SO}(3)\right\} .
$$

Among these groups, we can distinguish:

Planar groups: $\left\{\mathbb{1}, \mathrm{Z}_{n}, \mathrm{D}_{n}, \mathrm{SO}(2), \mathrm{O}(2)\right\}$, which are $\mathrm{O}(2)$-closed subgroups.

Exceptional groups: $\{\mathscr{T}, \mathcal{O}, \mathscr{I}, \mathrm{SO}(3)\}$, of which the first three are rotational symmetry groups of Platonic polyhedra.

Let us detail first the set of planar subgroups. We fix a base $(\mathbf{i} ; \mathbf{j} ; \mathbf{k})$ of $\mathbb{R}^{3}$, and denote by $\mathbf{Q}(\mathbf{v} ; \theta) \in \mathrm{SO}(3)$ the rotation about $\mathbf{v} \in \mathbb{R}^{3}$, with angle $\theta \in[0 ; 2 \pi)$.

- $\mathbb{1}$ is the identity.

- $\mathrm{Z}_{n}(n \geq 2)$ is the cyclic group of order $n$, generated by the $n$-fold rotation $\mathbf{Q}(\mathbf{k} ; \theta=2 \pi / n)$. which is the symmetry group of a chiral polygon.

- $\mathrm{D}_{n}(n \geq 2)$ is the dihedral group of order $2 n$ generated by $\mathrm{Z}_{n}$ and $\mathbf{Q}(\mathbf{i} ; \pi)$, which is the symmetry group of a regular polygon.

- $\mathrm{SO}(2)$ is the subgroup of rotations $\mathbf{Q}(\mathbf{k} ; \theta)$ with $\theta \in[0 ; 2 \pi)$.

- $\mathrm{O}(2)$ is the subgroup generated by $\mathrm{SO}(2)$ and $\mathbf{Q}(\mathbf{i} ; \pi)$.

The classes of exceptional subgroups are: $\mathscr{T}$, the tetrahedral group of order 12 which fixes a tetrahedron, $\mathbb{O}$, the octahedral group of order 24 which fixes an octahedron (or a cube), and $\mathscr{I}$, the subgroup of order 60 which fixes an icosahedron (or a dodecahedron).

In Section 6, the symmetry classes of $\mathbb{S}^{2 n}$ and $\mathbb{G}^{2 n}$ are obtained:

Lemma 2.2. The symmetry classes of $\mathbb{S}^{2 n}$ are:

$$
\begin{aligned}
\mathfrak{I}\left(\mathbb{S}^{2}\right) & =\left\{\left[\mathrm{D}_{2}\right],[\mathrm{O}(2)],[\mathrm{SO}(3)]\right\}, \\
\mathfrak{I}\left(\mathbb{S}^{4}\right)=\left\{[\mathbb{1}],\left[\mathrm{Z}_{2}\right],\left[\mathrm{D}_{2}\right],\left[\mathrm{D}_{3}\right],\left[\mathrm{D}_{4}\right],[\mathrm{O}(2)],[\mathrm{O}],[\mathrm{SO}(3)]\right\}, & \\
\mathfrak{I}\left(\mathbb{S}^{2 n}\right)=\left\{[\mathbb{1}],\left[\mathrm{Z}_{2}\right], \ldots,\left[\mathrm{Z}_{2(n-1)}\right],\left[\mathrm{D}_{2}\right], \ldots,\left[\mathrm{D}_{2 n}\right],[\mathrm{O}(2)],[\mathcal{T}],[\mathrm{O}],[\mathcal{F}],[\mathrm{SO}(3)]\right\}, & \text { if } n \geq 3 .
\end{aligned}
$$


Lemma 2.3. The symmetry classes of $\mathbb{G}^{2 n}$ are:

$$
\begin{aligned}
\mathfrak{I}\left(\mathbb{G}^{2}\right) & =\left\{[\mathbb{1}],\left[\mathrm{Z}_{2}\right],\left[\mathrm{D}_{2}\right],[\mathrm{SO}(2)],[\mathrm{O}(2)],[\mathrm{SO}(3)]\right\}, \\
\mathfrak{I}\left(\mathbb{G}^{4}\right) & =\left\{[\mathbb{1}],\left[\mathrm{Z}_{2}\right],\left[\mathrm{Z}_{3}\right],\left[\mathrm{Z}_{4}\right],\left[\mathrm{D}_{2}\right],\left[\mathrm{D}_{3}\right],\left[\mathrm{D}_{4}\right],[\mathrm{SO}(2)],[\mathrm{O}(2)],[\mathscr{T}],[\mathcal{O}],[\mathrm{SO}(3)]\right\}, \\
\mathfrak{I}\left(\mathbb{G}^{2 n}\right) & =\left\{[\mathbb{1}],\left[\mathrm{Z}_{2}\right], \ldots,\left[\mathrm{Z}_{2 n}\right],\left[\mathrm{D}_{2}\right], \ldots,\left[\mathrm{D}_{2 n}\right],[\mathrm{SO}(2)],[\mathrm{O}(2)],[\mathscr{T}],[\mathcal{O}],[\mathscr{F}],[\mathrm{SO}(3)]\right\}, \\
& \text { if } n \geq 3 .
\end{aligned}
$$

The following table lists how many classes there are for each $n$ :

\begin{tabular}{|rrrc|}
\hline$n=$ & 1 & 2 & $\geq 3$ \\
$\# \Im\left(\mathbb{S}^{2 n}\right)$ & 3 & 8 & $2(2 n+1)$ \\
$\# \Im\left(\mathbb{G}^{2 n}\right)$ & 6 & 12 & $4 n+5$ \\
\hline
\end{tabular}

The symmetry classes of $\mathbb{T}^{2 n}$ are clarified by the following theorem:

Theorem I. Let $\mathbb{T}^{2 n}$ be a tensor space. Then either $\mathfrak{I}\left(\mathbb{T}^{2 n}\right)=\mathfrak{I}\left(\mathbb{S}^{2 n}\right)$ or $\mathfrak{I}\left(\mathbb{T}^{2 n}\right)=$ $\Im\left(\mathbb{G}^{2 n}\right)$.

In other words, the number and type of classes are the same as those of

- $\mathbb{S}^{2 n}$, the space of $2 n$-order completely symmetric tensors, in which case the number of classes is minimal, or

- $\mathbb{G}^{2 n}$, the space of $2 n$-order generic tensors, in which case the number of classes is maximal.

In fact, as specified by the following theorems, in most situations the number of classes is indeed maximal.

Theorem II (coupling tensors). Let us consider $\mathbb{T}^{2 p}$ the space of coupling tensors between two physics described by two tensor vector spaces $\mathbb{E}_{1}$ and $\mathbb{E}_{2}$. If these tensor spaces are of orders greater than or equal to one, then $\Im\left(\mathbb{T}^{2 p}\right)=\Im\left(\mathbb{G}^{2 p}\right)$.

Theorem III (proper tensors). Let us consider $\mathbb{T}^{2 p}$, the space of tensors of a proper physics described by the tensor vector space $\mathbb{E}$. If this tensor space is of order $p \geq 3$, and is solely defined in terms of its index symmetries, then $\mathfrak{I}\left(\mathbb{T}^{2 p}\right)=\mathfrak{I}\left(\mathbb{G}^{2 p}\right)$.

Remark 2.4. Exceptions occur for

$p=1$, when the space of symmetric second-order tensors is obtained, and $p=2$, when, in the case of $\mathbb{T}_{(i j)}$, the space of elasticity tensors is obtained.

In each of these situations the number of classes is minimal. There is no other situation where this case occurs. It should therefore be concluded that the space of elasticity tensors is exceptional. 
Second strain-gradient elasticity (SSGE). Application of the former theorems will be made on the even-order tensors of SSGE. First, the constitutive equations will be summed up, and then the results will be stated. It worth noting that obtaining the same results with the Forte-Vianello approach would have been far more complicated.

Constitutive laws. In the second strain-gradient theory of linear elasticity [Mindlin 1965; Forest et al. 2011], the constitutive law gives the symmetric Cauchy stress tensor ${ }^{10} \sigma^{(2)}$ and the hyperstress tensors $\tau^{(3)}$ and $\omega^{(4)}$ in terms of the infinitesimal strain tensor $\varepsilon^{(2)}$ and its gradients $\eta^{(3)}=\varepsilon^{(2)} \otimes \nabla$ and $\kappa^{(4)}=\varepsilon^{(2)} \otimes \nabla \otimes \nabla$ through the three linear relations:

$$
\begin{aligned}
\sigma^{(2)} & =\mathbf{E}^{(4)}: \varepsilon^{(2)}+\mathbf{M}^{(5)} \therefore \eta^{(3)}+\mathbf{N}^{(6)}:: \kappa^{(4)}, \\
\tau^{(3)} & =\mathbf{M}^{T(5)}: \varepsilon+\mathbf{A}^{(6)} \therefore \eta^{(3)}+\mathbf{O}^{(7)}:: \kappa^{(4)}, \\
\omega^{(4)} & =\mathbf{N}^{T(6)}: \varepsilon^{(2)}+\mathbf{O}^{T(7)} \therefore \eta^{(3)}+\mathbf{B}^{(8)}:: \kappa^{(4)},
\end{aligned}
$$

where $:, \therefore$, and :: denote, respectively, the double, third, and fourth contracted products. Above, ${ }^{11} \sigma_{(i j)}, \varepsilon_{(i j)}, \tau_{(i j) k}, \eta_{(i j) k}=\varepsilon_{(i j), k}, \omega_{(i j)(k l)}$, and $\kappa_{(i j)(k l)}=\varepsilon_{(i j),(k l)}$ are, respectively, the matrix components of $\sigma^{(2)}, \varepsilon^{(2)}, \tau^{(3)}, \eta^{(3)}, \omega^{(4)}$, and $\kappa^{(4)}$ relative to an orthonormal basis $(\mathbf{i} ; \mathbf{j} ; \mathbf{k})$ of $\mathbb{R}^{3}$. And $E_{(i j)} \frac{(l m)}{}, M_{(i j)(l m) n}, N_{(i j)(k l)(m n)}$, $A_{\underline{(i j) k} \underline{(l m) n},}, O_{(i j) k(l m)(n o)}$, and $B_{(i j)(k l)} \underline{(m n)(o p)}$ are the matrix components of the related elastic stiffness tensors.

Symmetry classes. The symmetry classes of the elasticity tensors and of the first strain-gradient elasticity tensors has been studied in [Forte and Vianello 1996; Le Quang et al. 2012]. Hence, here we solely consider the spaces of coupling tensors $\mathbf{N}^{(6)}$ and of second strain-gradient elasticity tensors $\mathbf{B}^{(8)}$.

- We define $\mathbb{C}$ es to be the space of coupling tensors between classical elasticity and second strain-gradient elasticity:

$$
\mathbb{C} e s=\left\{\mathbf{N}^{(6)} \in \mathbb{G}^{6} \mid N_{(i j)(k l)(m n)}\right\} .
$$

A direct application of Theorem II leads to the result that $\Im(\mathbb{C} e s)=\left\{[\mathbb{1}],\left[\mathrm{Z}_{2}\right], \ldots,\left[\mathrm{Z}_{6}\right],\left[\mathrm{D}_{2}\right], \ldots,\left[\mathrm{D}_{6}\right],[\mathrm{SO}(2)],[\mathrm{O}(2)],[\mathscr{T}],[\mathrm{O}],[\mathscr{g}],[\mathrm{SO}(3)]\right\}$

Therefore $\mathbb{C}$ es is divided into 17 symmetry classes.

- We define Sgr to be the space of second strain-gradient elasticity tensors:

$$
\operatorname{Sgr}=\left\{\mathbf{O}^{(8)} \in \mathbb{G}^{8} \mid O_{\underline{(i j)(k l)}} \underline{(m n)(o p)}\right\} .
$$

${ }^{10}$ In this subsection only, tensor orders will be indicated by superscripts in parentheses.

${ }^{11}$ The comma classically indicates the partial derivative with respect to spatial coordinates. Superscript $T$ denotes transposition. Transposition is defined by permuting the $p$ first indices with the $q$ last, where $p$ is the tensorial order of the image of a $q$-order tensor. 
A direct application of Theorem III leads to the result that $\mathfrak{I}(\operatorname{Sgr})=\left\{[\mathbb{1}],\left[\mathrm{Z}_{2}\right], \ldots,\left[\mathrm{Z}_{8}\right],\left[\mathrm{D}_{2}\right], \ldots,\left[\mathrm{D}_{8}\right],[\mathrm{SO}(2)],[\mathrm{O}(2)],[\mathscr{T}],[\mathrm{O}],[\mathscr{F}],[\mathrm{SO}(3)]\right\}$

Therefore Sgr is divided into 21 symmetry classes.

\section{Mathematical framework}

In this section the mathematical framework of symmetry analysis is introduced. In the first two subsections the notions of symmetry group and class are introduced; the last is devoted to the introduction of irreducible spaces. The presentation is rather general, and will be specialized to tensor spaces only at the end of the section.

Isotropy/symmetry groups. Let $\rho$ be a representation of a compact real Lie group ${ }^{12}$ $G$ on a finite dimensional $\mathbb{R}$-linear space $\mathscr{E}$ :

$$
\rho: G \rightarrow \mathrm{GL}(\mathscr{E}) .
$$

This action will also be denoted by

$$
g \cdot \mathbf{x}=\rho(g)(\mathbf{x}),
$$

where $g \in G$ and $\mathbf{x} \in \mathscr{E}$. For any element of $\mathscr{E}$, the set of operations $g$ in $G$ leaving this element invariant is defined as

$$
\Sigma_{\mathbf{x}}:=\{g \in G \mid g \cdot \mathbf{x}=\mathbf{x}\} .
$$

This set is known to physicists as the symmetry group of $\mathbf{x}$ and to mathematicians as the stabilizer or isotropy subgroup of $\mathbf{x}$. Owing to $G$-compactness, every isotropy subgroup is a closed subgroup of $G$. Conversely, a dual notion can be defined for $G$-elements. For any subgroup $K$ of $G$, the set of $K$-invariant elements in $\mathscr{E}$ is defined as

$$
\mathscr{E}^{K}:=\{\mathbf{x} \in \mathscr{E} \mid k \cdot \mathbf{x}=\mathbf{x} \text { for all } k \in K\}
$$

Such a set is referred to as a fixed point set and is a linear subspace of $\mathscr{E}$. In this context we will set $\mathrm{d}(K)=\operatorname{dim} \mathscr{E}^{K}$. It has to be observed that fixed-point sets are group inclusion reversing, that is, for subgroups $K_{1}$ and $K_{2}$ of $G$, we have the property

$$
K_{1} \subset K_{2} \Longrightarrow \mathscr{E}^{K_{2}} \subset \mathscr{E}^{K_{1}}
$$

For a given isotropy group $K$, the former sets are linked by the property

$$
\mathbf{x} \in \mathscr{E}^{\mathscr{E}} K \Longrightarrow K \subset \Sigma_{\mathbf{x}}
$$

${ }^{12}$ In the following $G$ will always represent a compact real Lie group, so this specification will mostly be omitted. 
Isotropy/symmetry classes. We aim to describe objects that have the same symmetry properties but may differ by their orientations in space. The first point is to define the set of all the positions an object can have. To that aim we consider the $G$-orbit of an element $\mathbf{x}$ of $\mathscr{E}$ :

$$
\operatorname{Orb}(\mathbf{x}):=\{g \cdot \mathbf{x} \mid g \in G\} \subset \mathscr{E} .
$$

Due to $G$-compactness this set is a submanifold of $\mathscr{E}$. Elements of $\operatorname{Orb}(\mathbf{x})$ will be said to be $G$-related. A fundamental observation is that $G$-related vectors have conjugate symmetry groups. More precisely, ${ }^{13}$

$$
\operatorname{Orb}(\mathbf{x})=\operatorname{Orb}(\mathbf{y}) \Longrightarrow \Sigma_{\mathbf{x}}=g \Sigma_{\mathbf{y}} g^{-1} \text { for some } g \in G \text {. }
$$

Let us define the conjugacy class of a subgroup $K \subset G$ by

$$
[K]=\left\{K^{\prime} \subset G \mid K^{\prime}=g K g^{-1} \text { for some } g \in G\right\} .
$$

An isotropy class (or symmetry class) $[\Sigma]$ is defined as the conjugacy class of an isotropy subgroup $\Sigma$. This definition implies that there exists a vector $\mathbf{x} \in \mathscr{E}$ such that $\Sigma=\Sigma_{\mathbf{X}}$ and $\Sigma^{\prime} \in[\Sigma]$; furthermore $\Sigma^{\prime}=g \Sigma g^{-1}$ for some $g \in G$. The notion of isotropy class is a good notion to define the symmetry property of an object modulo its orientation: a symmetric group is related to a specific vector, but we deal with orbits, which are related to isotropy classes because of (3-1). Due to $G$ compactness there is only a finite number of isotropy classes [Bredon 1972], and we introduce the notation

$$
\Im(\mathscr{I}):=\left\{[\mathbb{1}] ;\left[\Sigma_{1}\right] ; \ldots ;\left[\Sigma_{l}\right]\right\},
$$

the set of all isotropy classes. In the case $G=\mathrm{SO}(3)$ this result is known as Hermann's theorem [Herman 1945; Auffray 2008]. The elements of $\mathfrak{I}(\mathscr{E})$ are conjugate to $\mathrm{SO}$ (3)-closed subgroups; this collection was introduced in Lemma 2.1.

Irreducible spaces. For every linear subspace $\mathscr{F}$ of $\mathscr{E}$, we set

$$
g \cdot \mathscr{F}:=\{g \cdot \mathbf{x} \mid g \in G, \mathbf{x} \in \mathscr{F}\}
$$

and we say that $\mathscr{F}$ is $G$-stable if $g \cdot \mathscr{F} \subset \mathscr{F}$ for every $g \in G$. It is clear that, for every representation, the subspaces $\{0\}$ and $\mathscr{E}$ are always $G$-stable. If, for a representation $\rho$ on $\mathscr{E}$, the only $G$-invariant spaces are the proper ones, the representation will be said to be irreducible. For a compact Lie group, the Peter-Weyl theorem [Sternberg 1994] ensures that every representation can be split into a direct sum of irreducible

\footnotetext{
${ }^{13}$ With the classical coset notation, if $H$ is a subgroup of $G$ and $g \in G$ is not in the subgroup $H$, then a left coset of $H$ in $G$ is defined

$$
g H=\{g h: h \in H\},
$$
}

and symmetrically for a right coset. 
ones. Furthermore, in the case $G=\mathrm{SO}(3)$, those irreducible representations are explicitly known.

There is a natural action of $\mathrm{SO}(3)$ on the space of $\mathbb{R}^{3}$-harmonic polynomials. If $p$ is a harmonic polynomial and $\mathbf{x} \in \mathbb{R}^{3}$, then for every $g \in \mathrm{SO}$ (3) we write

$$
g \cdot p(\mathbf{x})=p\left(g^{-1} \cdot \mathbf{x}\right) .
$$

Harmonic polynomials form a graded vector space, and to each subspace of a given degree is associated an $\mathrm{SO}(3)$-irreducible representation. $\mathscr{H}^{k}$ will be the vector space of harmonic polynomials of degree $k$, with $\operatorname{dim} \mathscr{H}^{k}=2 k+1$. If we take a vector space $V$ to be an $\mathrm{SO}(3)$-representation, it can be decomposed into $\mathrm{SO}(3)$-irreducible spaces:

$$
V=\bigoplus \mathscr{H}^{k_{i}}
$$

Grouping together irreducible spaces of the same order, one obtains the $\mathrm{SO}(3)$ isotypic decomposition of a representation:

$$
V=\bigoplus_{i=0}^{n} \alpha_{i} \mathscr{H}^{i},
$$

where $\alpha_{i}$ is the multiplicity of the irreducible space $\mathscr{H}^{i}$ in the decomposition, and $n$ is the order of the highest-order irreducible space of the decomposition.

Application to tensor spaces. In mechanics, $V$ is a vector subspace of $\bigotimes^{p} \mathbb{R}^{3}$. In $\mathbb{R}^{3}$ there exists an isomorphism, $\phi$, between harmonic polynomial spaces and harmonic tensor spaces [Backus 1970; Forte and Vianello 1996]. Therefore all that has been said for harmonic polynomials can be translated in terms of harmonic tensors. A detailed discussion on this isomorphism can be found in [Backus 1970]. Therefore $\mathbb{H}^{k}=\varphi\left(\mathscr{H}^{k}\right)$ is the space of harmonic tensors, that is, the space of completely symmetric and traceless tensors. According to this isomorphism, any tensor space $\mathbb{T}^{n}$ can be decomposed into $\mathrm{SO}(3)$-irreducible tensors:

$$
\mathbb{T}^{n}=\bigoplus_{i=0}^{n} \alpha_{i} \boxplus^{i} .
$$

The symmetry group of $\mathbf{T} \in \mathbb{T}^{n}$ is the intersection of the symmetry groups of all its harmonic components: ${ }^{14}$

$$
\Sigma_{\mathbf{T}}=\bigcap_{i=0}^{n}\left(\bigcap_{j=0}^{\alpha_{i}} \Sigma_{\mathrm{H}^{i, j}}\right) .
$$

${ }^{14}$ In the notation $\mathrm{H}^{i, j}$, the first superscript refers to the order of the harmonic tensor, while the second indexes the multiplicity of $\mathrm{H}^{i}$ in the decomposition. 
In the same way, $\Im\left(\mathbb{T}^{n}\right)$ will be obtained as a function of the symmetry classes of the irreducible representations involved in the harmonic decomposition of $\mathbb{T}^{n}$. The symmetry classes of $\mathrm{SO}(3)$-irreducible representations are explicitly known [Ihrig and Golubitsky 1984; Golubitsky et al. 1988]; what is unknown is how to combine these results to determine the symmetry classes of $V$ (or $\mathbb{T}^{n}$ ).

\section{The clips operation}

The aim of this section is to construct symmetry classes of a reducible representation from irreducible ones. With that goal a new class-operator, named the clips operator, will be defined. The main result of this section is given in Table 2, which contains all clips operations between $\mathrm{SO}(3)$-closed subgroups. It is worth noting that this table contains more results than strictly needed for the proofs of our theorems. Nevertheless, we believe that these results are interesting on their own and may find application in other problems. The explicit proofs of these results can be found in the Appendix.

Here we consider the intersection of two symmetry classes only. Extensions to more general reducible representations will be treated in Section 5. Let us start with the following lemma:

Lemma 4.1. Let $\mathscr{E}$ be a representation of a compact Lie group $G$ that splits into a direct sum of two $G$-stable subspaces:

$$
\mathscr{E}=\mathscr{E}_{1} \oplus \mathscr{E}_{2} \text {, where } g \cdot \mathscr{E}_{1} \subset \mathscr{E}_{1} \text { and } g \cdot \mathscr{E}_{2} \subset \mathscr{E}_{2} \text { for all } g \in G \text {. }
$$

If we denote by $\mathfrak{I}$ the set of all isotropy classes associated with $\mathscr{E}$ and by $\mathfrak{I}_{i}$ the set of all isotropy classes associated with $\mathscr{E}_{i}(i=1,2)$, then $[\Sigma] \in \mathfrak{I}$ if and only if there exist $\left[\Sigma_{1}\right] \in \mathfrak{I}_{1}$ and $\left[\Sigma_{2}\right] \in \mathfrak{I}_{2}$ such that $\Sigma=\Sigma_{1} \cap \Sigma_{2}$.

Proof. If we take $\left[\Sigma_{1}\right] \in \mathfrak{I}_{1}$ and $\left[\Sigma_{2}\right] \in \mathfrak{I}_{2}$, we know there exist two vectors $\mathbf{x}_{1} \in \mathscr{E}_{1}$ and $\mathbf{x}_{2} \in \mathscr{E}_{2}$ such that $\Sigma_{i}=\Sigma_{\mathbf{x}_{i}}(i=1,2)$. Then, let $\mathbf{x}:=\mathbf{x}_{1}+\mathbf{x}_{2}$.

For every $g \in \Sigma_{1} \cap \Sigma_{2}$ we have $g \cdot \mathbf{x}_{1}+g \cdot \mathbf{x}_{2}=\mathbf{x}_{1}+\mathbf{x}_{2}=\mathbf{x}$; thus $\Sigma_{1} \cap \Sigma_{2} \subset \Sigma_{\mathbf{x}}$. Conversely for every $g \in \Sigma_{\mathbf{x}}$ we have

$$
g \cdot \mathbf{x}=\mathbf{x}=g \cdot \mathbf{x}_{1}+g \cdot \mathbf{x}_{2} .
$$

But, since the $\mathscr{E}_{i}$ are $G$-stable and form a direct sum, we conclude that $g \cdot \mathbf{x}_{i}=\mathbf{x}_{i}$ $(i=1,2)$. The reverse inclusion is proved.

The other implication is similar: if we take $[\Sigma] \in \mathfrak{I}$ then we have $\Sigma=\Sigma_{\mathbf{x}}$ for some $\mathbf{x} \in \mathscr{E}$, and $\mathbf{x}$ can be decomposed into $\mathbf{x}_{1}+\mathbf{x}_{2}$. The same proof as above shows that $\Sigma=\Sigma_{\mathbf{x}_{1}} \cap \Sigma_{\mathbf{x}_{2}}$.

Lemma 4.1 shows that the isotropy classes of a direct sum are related to intersections of isotropy subgroups. But as intersection of classes is meaningless, the 
results cannot be directly extended. To solve this problem, a tool called the clips operator will be introduced. We will make sure of a lemma:

Lemma 4.2. For every two $G$-classes $\left[\Sigma_{i}\right](i=1,2)$, and for every $g_{1}$ and $g_{2}$ in $G$, there exists $g=g_{1}^{-1} g_{2}$ in $G$ such that

$$
\left[g_{1} \Sigma_{1} g_{1}^{-1} \cap g_{2} \Sigma_{2} g_{2}^{-1}\right]=\left[\Sigma_{1} \cap g \Sigma_{2} g^{-1}\right] .
$$

Proof. Let $g=g_{1}^{-1} g_{2}$ and

$$
\Sigma=g_{1} \Sigma_{1} g_{1}^{-1} \cap g_{2} \Sigma_{2} g_{2}^{-1} .
$$

For every $\gamma \in \Sigma$ we have $\gamma=g_{1} \gamma_{1} g_{1}^{-1}=g_{2} \gamma_{2} g_{2}^{-1}$ for some $\gamma_{i} \in \Sigma_{i}(i=1,2)$; then

$$
g_{1} \gamma g_{1}^{-1}=\gamma_{1} \in \Sigma_{1} \text { and } g_{1} \gamma g_{1}^{-1}=g \gamma_{2} g^{-1} \in g \Sigma_{2} g^{-1} \text {. }
$$

Thus we have $g_{1} \Sigma g_{1}^{-1} \subset \Sigma_{1} \cap g \Sigma_{2} g^{-1}$, and conversely. Since $g_{1} \Sigma g_{1}^{-1}$ is conjugate to $\Sigma$, we have proved the lemma.

Definition 4.3 (clips operator). We define the action of the clips operator $\odot$ on $G$-classes $\left[\Sigma_{1}\right]$ and $\left[\Sigma_{2}\right]$ by setting

$$
\left[\Sigma_{1}\right] \odot\left[\Sigma_{2}\right]:=\left\{\left[\Sigma_{1} \cap g \Sigma_{2} g^{-1}\right] \mid g \in G\right\},
$$

which is a subset of $G$-classes.

If we denote by $\mathbb{1}$ the identity subgroup, we have some immediate properties:

Proposition 4.4. For every $G$-class $[\Sigma]$ we have

$$
[\mathbb{1}] \odot[\Sigma]=\{[\mathbb{1}]\} \text { and }[G] \odot[\Sigma]=\{[\Sigma]\} \text {. }
$$

Given two $G$-representations $\mathscr{E}_{1}$ and $\mathscr{E}_{2}$, if we denote by $\mathfrak{I}_{i}$ the set of all isotropy classes of $\mathscr{E}_{i}$, the action of the clips operator can be extended to these sets via

$$
\mathfrak{I}_{1} \odot \mathfrak{I}_{2}:=\bigcup_{\substack{\Sigma_{1} \in \mathfrak{I}_{1} \\ \Sigma_{2} \in \mathfrak{I}_{2}}}\left[\Sigma_{1}\right] \odot\left[\Sigma_{2}\right] .
$$

Then, by Lemma 4.1, we obtain:

Corollary 4.5. For every two G-representations $\mathscr{E}_{1}$ and $\mathscr{E}_{2}$, if $\mathfrak{I}_{1}$ denotes the isotropy classes of $\mathscr{E}_{1}$ and $\mathfrak{I}_{2}$ the isotropy classes of $\mathscr{E}_{2}$, then $\mathfrak{I}_{1} \odot \mathfrak{I}_{2}$ are all the isotropy classes of $\mathscr{E}_{1} \oplus \mathscr{E}_{2}$.

Theorem 4.6. For any two $\mathrm{SO}(3)$-closed subgroups $\Sigma_{1}$ and $\Sigma_{2}$, we have $\mathbb{1} \in$ $\left[\Sigma_{1}\right] \odot\left[\Sigma_{2}\right]$. The remaining classes in the clips product $\left[\Sigma_{1}\right] \odot\left[\Sigma_{2}\right]$ are given in Table 2. 


\begin{tabular}{|c|c|c|c|c|c|c|c|}
\hline () & {$\left[\mathrm{Z}_{n}\right]$} & {$\left[\mathrm{D}_{n}\right]$} & {$[\mathscr{T}]$} & [0] & {$[\mathscr{I}]$} & {$[\mathrm{SO}(2)]$} & {$[\mathrm{O}(2)]$} \\
\hline$\left[\mathrm{Z}_{m}\right]$ & {$\left[\mathrm{Z}_{d}\right]$} & & & & & & \\
\hline$\left[\mathrm{D}_{m}\right]$ & $\begin{array}{l}{\left[\mathrm{Z}_{d_{2}}\right]} \\
{\left[\mathrm{Z}_{d}\right]}\end{array}$ & $\begin{array}{c}{\left[\mathrm{Z}_{d_{2}}\right]} \\
{\left[\mathrm{Z}_{d^{\prime}}\right],\left[\mathrm{Z}_{d z}\right]} \\
{\left[\mathrm{Z}_{d}\right],\left[\mathrm{D}_{d}\right]}\end{array}$ & & & & & \\
\hline$[\mathscr{T}]$ & $\begin{array}{l}{\left[\mathrm{Z}_{d_{2}}\right]} \\
{\left[\mathrm{Z}_{d_{3}}\right]}\end{array}$ & $\begin{array}{c}{\left[\mathrm{Z}_{2}\right]} \\
{\left[\mathrm{Z}_{d_{3}}\right],\left[\mathrm{D}_{d_{2}}\right]}\end{array}$ & $\begin{array}{l}{\left[\mathrm{Z}_{2}\right]} \\
{\left[\mathrm{Z}_{3}\right]} \\
{[\mathscr{T}]}\end{array}$ & & & & \\
\hline$[0]$ & $\begin{array}{l}{\left[\mathrm{Z}_{d_{2}}\right]} \\
{\left[\mathrm{Z}_{d_{3}}\right]} \\
{\left[\mathrm{Z}_{d_{4}}\right]}\end{array}$ & $\begin{array}{c}{\left[\mathrm{Z}_{2}\right]} \\
{\left[\mathrm{Z}_{d_{3}}\right],\left[\mathrm{Z}_{d_{4}}\right]} \\
{\left[\mathrm{D}_{d_{2}}\right],\left[\mathrm{D}_{d_{3}}\right]} \\
{\left[\mathrm{D}_{d_{4}}\right]}\end{array}$ & $\begin{array}{l}{\left[\mathrm{Z}_{2}\right]} \\
{\left[\mathrm{Z}_{3}\right]} \\
{[\mathscr{T}]}\end{array}$ & $\begin{array}{c}{\left[\mathrm{Z}_{2}\right]} \\
{\left[\mathrm{D}_{2}\right],\left[\mathrm{Z}_{3}\right]} \\
{\left[\mathrm{D}_{3}\right],\left[\mathrm{Z}_{4}\right]} \\
{\left[\mathrm{D}_{4}\right],[\mathrm{O}]}\end{array}$ & & & \\
\hline$[\mathscr{I}]$ & $\begin{array}{l}{\left[\mathrm{Z}_{d_{2}}\right]} \\
{\left[\mathrm{Z}_{d_{3}}\right]} \\
{\left[\mathrm{Z}_{d_{5}}\right]}\end{array}$ & $\begin{array}{c}{\left[\mathrm{Z}_{2}\right]} \\
{\left[\mathrm{Z}_{d_{3}}\right],\left[\mathrm{Z}_{d_{5}}\right]} \\
{\left[\mathrm{D}_{d_{2}}\right]} \\
{\left[\mathrm{D}_{d_{3}}\right],\left[\mathrm{D}_{d_{5}}\right]}\end{array}$ & $\begin{array}{l}{\left[\mathrm{Z}_{2}\right]} \\
{\left[\mathrm{Z}_{3}\right]} \\
{[\mathscr{T}]}\end{array}$ & $\begin{array}{c}{\left[\mathrm{Z}_{2}\right]} \\
{\left[\mathrm{Z}_{3}\right],\left[\mathrm{D}_{3}\right]} \\
{[\mathscr{T}]}\end{array}$ & $\begin{array}{c}{\left[\mathrm{Z}_{2}\right]} \\
{\left[\mathrm{Z}_{3}\right],\left[\mathrm{D}_{3}\right]} \\
{\left[\mathrm{Z}_{5}\right],\left[\mathrm{D}_{5}\right]} \\
{[\mathscr{\Phi}]}\end{array}$ & & \\
\hline$[\mathrm{SO}(2)]$ & {$\left[\mathrm{Z}_{n}\right]$} & $\begin{array}{l}{\left[\mathrm{Z}_{2}\right]} \\
{\left[\mathrm{Z}_{n}\right]}\end{array}$ & $\begin{array}{l}{\left[Z_{2}\right]} \\
{\left[Z_{3}\right]}\end{array}$ & $\begin{array}{c}{\left[\mathrm{Z}_{2}\right]} \\
{\left[\mathrm{Z}_{3}\right],\left[\mathrm{Z}_{4}\right]}\end{array}$ & $\begin{array}{c}{\left[\mathrm{Z}_{2}\right]} \\
{\left[\mathrm{Z}_{3}\right],\left[\mathrm{Z}_{5}\right]}\end{array}$ & {$[\mathrm{SO}(2)]$} & \\
\hline$[\mathrm{O}(2)]$ & $\begin{array}{c}{\left[\mathrm{Z}_{d_{2}}\right]} \\
{\left[\mathrm{Z}_{n}\right]}\end{array}$ & $\begin{array}{l}{\left[\mathrm{Z}_{2}\right]} \\
{\left[\mathrm{D}_{n}\right]}\end{array}$ & $\begin{array}{l}{\left[\mathrm{D}_{2}\right]} \\
{\left[\mathrm{Z}_{3}\right]}\end{array}$ & $\begin{array}{c}{\left[\mathrm{D}_{2}\right]} \\
{\left[\mathrm{D}_{3}\right],\left[\mathrm{D}_{4}\right]}\end{array}$ & $\begin{array}{c}{\left[\mathrm{D}_{2}\right]} \\
{\left[\mathrm{D}_{3}\right],\left[\mathrm{D}_{5}\right]}\end{array}$ & $\begin{array}{c}{\left[\mathrm{Z}_{2}\right]} \\
{[\mathrm{SO}(2)]}\end{array}$ & $\begin{array}{c}{\left[\mathrm{Z}_{2}\right]} \\
{[\mathrm{O}(2)]}\end{array}$ \\
\hline
\end{tabular}

Table 2. Action of the clips operation on $\mathrm{SO}(3)$-subgroups.

Conventions: $\mathrm{Z}_{1}:=\mathrm{D}_{1}:=\mathbb{1} ; d_{2}:=\operatorname{gcd}(n, 2) ; d_{3}:=\operatorname{gcd}(n, 3)$; $d_{5}:=\operatorname{gcd}(n, 5) ; d_{2}^{\prime}:=\operatorname{gcd}(m, 2) ; d z:=2$ if $d=1,1$ otherwise; $d_{4}:=4$ if $4 \mid n, 1$ otherwise.

\section{Isotropy classes of harmonic tensors}

We now turn to the construction of the symmetry classes of a reducible representation from its irreducible components. The first subsection states the main results on symmetry classes of irreducible representations. Thereafter we derive from the results of the previous section the basic properties of reducible representations. These results will be used in Section 6 to prove the theorems stated in Section 2.

From now on, all results will be expressed in terms of tensor spaces.

Isotropy classes of irreducibles. The following result was obtained in [Ihrig and Golubitsky 1984; Golubitsky et al. 1988]: 
Theorem 5.1. Let $\mathrm{SO}(3)$ act on $\mathbb{U}^{k}$. The following groups are symmetry classes of $\mathbb{\boxplus}^{k}$ :

(a) $\mathbb{1}$ for $k \geq 3$.

(b) $\mathrm{Z}_{n}(n \geq 2)$ for $n \leq k$ when $k$ is odd, $n \leq k / 2$ when $k$ is even.

(c) $\mathrm{D}_{n}(n \geq 2)$ for $n \leq k$.

(d) $\mathscr{T}$ for $k=3,6,7$ or $k \geq 9$.

(e) o for $k \neq 1,2,3,5,7,11$.

(f) $\mathscr{I}$ for $k=6,10,12,15,18$ or $k \geq 20$ and $k \neq 23,29$.

(g) $\mathrm{SO}(2)$ for $k$ odd.

(h) $\mathrm{O}(2)$ for $k$ even.

(i) $\mathrm{SO}(3)$ for any $k$.

For future purposes, let us introduce some notation. For each integer $k$, we let:

$$
\begin{aligned}
\Gamma_{\mathscr{T}(k)} & := \begin{cases}\mathscr{T} & \text { if } \mathscr{T} \in \mathfrak{I}^{k}, \\
\varnothing & \text { otherwise; }\end{cases} \\
\Gamma_{\mathscr{O}(k)} & := \begin{cases}\mathcal{O} & \text { if } \mathcal{O} \in \mathfrak{I}^{k}, \\
\varnothing & \text { otherwise; }\end{cases} \\
\Gamma_{\mathscr{I}(k)} & := \begin{cases}\mathscr{I} & \text { if } \mathscr{I} \in \mathfrak{I}^{k}, \\
\varnothing & \text { otherwise; }\end{cases} \\
\Sigma(k) & := \begin{cases}\mathrm{SO}(2) & \text { if } \mathrm{SO}(2) \in \mathfrak{I}^{k}, \\
\varnothing & \text { otherwise; }\end{cases} \\
\Omega(k) & := \begin{cases}\mathrm{O}(2) & \text { if } \mathrm{O}(2) \in \mathfrak{I}^{k}, \\
\varnothing & \text { otherwise; }\end{cases}
\end{aligned}
$$

where $\mathfrak{I}^{k}$ is the set of symmetry classes of $\mathbb{\sharp}^{k}$.

Isotropy classes of direct sum. We have this obvious lemma, directly deduced from Theorem 5.1:

Lemma 5.2.

$$
\begin{array}{ll}
\Gamma_{\mathscr{T}(k)} \neq \varnothing \Longrightarrow \quad\left\{\left[\mathrm{D}_{2}\right],\left[\mathrm{D}_{3}\right]\right\} \subset \mathfrak{I}^{k}, \\
\Gamma_{\mathscr{O}(k)} \neq \varnothing \Longrightarrow\left\{\left[\mathrm{D}_{2}\right],\left[\mathrm{D}_{3}\right],\left[\mathrm{D}_{4}\right]\right\} \subset \mathfrak{I}^{k}, \\
\Gamma_{\mathscr{I}(k)} \neq \varnothing \Longrightarrow\left\{\left[\mathrm{D}_{2}\right], \ldots,\left[\mathrm{D}_{5}\right]\right\} \subset \mathfrak{I}^{k} .
\end{array}
$$

We denote by $\mathfrak{I}(k, n)$ the $(n-1)$-fold self clips product of $\mathfrak{I}^{k}$, which is the set of isotropy classes of a $n$-tuple of $k$-th harmonic tensors [Auffray et al. 2011], that is, $n \mathbb{\natural}^{k}$. The basic operations are, for all integers $k \geq 1$ and $n \geq 2$,

$$
\mathfrak{I}(k, n):=\mathfrak{I}^{k} \odot \Im(k, n-1) \quad \text { and } \quad \mathfrak{I}(k, 1):=\mathfrak{I}^{k} \text {. }
$$


On the simple example of $\mathbb{H}^{2}$, the following fact can be observed:

$$
\mathfrak{I}(2, n):=\mathfrak{I}^{2} \odot \Im(2, n-1)=\mathfrak{I}^{2} \odot \mathfrak{I}^{2}=\left\{[\mathbb{1}],\left[\mathrm{Z}_{2}\right],\left[\mathrm{D}_{2}\right],[\mathrm{O}(2)],[\mathrm{SO}(3)]\right\} .
$$

This result can be generalized:

Corollary 5.3. For all integers $n \geq 2$ and $k \geq 2$, the isotropy classes of $n \mathbb{\boxplus}^{k}$ are

$$
\begin{aligned}
\mathfrak{I}(k, n)= & \mathfrak{I}^{k} \odot \mathfrak{I}^{k} \\
= & \left\{[\mathbb{1}],\left[\mathrm{Z}_{2}\right], \ldots,\left[\mathrm{Z}_{k}\right],\right. \\
& \left.\quad\left[\mathrm{D}_{2}\right], \ldots,\left[\mathrm{D}_{k}\right],\left[\Gamma_{\mathscr{T}(k)}\right],\left[\Gamma_{\mathscr{O}(k)}\right],\left[\Gamma_{\mathscr{I}(k)}\right],[\Sigma(k)],[\Omega(k)],[\operatorname{SO}(3)]\right\} .
\end{aligned}
$$

Proof. From Theorem 5.1 we know that $\left[\mathrm{D}_{l}\right] \in \mathfrak{I}^{k}$ for $2 \leq l \leq k$; furthermore, $[\mathrm{SO}(3)] \in \mathfrak{I}^{k}$. Then from Proposition 4.4 we know that, for all integers $2 \leq l \leq k$ we will have (by induction), for all $n \geq 2,\left[\mathrm{D}_{l}\right] \in \mathfrak{I}(k, n)$. Then, when we compute $\Im(k, n) \odot \mathfrak{I}^{k}$ we will have $\left[\mathrm{D}_{l}\right] \odot\left[\mathrm{D}_{l}\right]=\left\{[\mathbb{1}],\left[\mathrm{Z}_{l}\right],\left[\mathrm{D}_{l}\right]\right\}$. Neither $[\mathrm{O}(2)]$ nor $[\mathrm{SO}(2)]$, with cyclic or dihedral conjugacy classes, generates other cases. The same occurs for the clips product of cyclic groups. Now, because of Lemma 5.2 we also see that no exceptional conjugacy class generates other cases.

Corollary 5.4. For all integers $2 \leq 2 p<2 q$, we have

$$
\begin{aligned}
& \mathfrak{I}(2 p, 2 q):= \mathfrak{I}^{2 p} \odot \mathfrak{I}^{2 q} \\
&=\left\{[\mathbb{1}],\left[\mathrm{Z}_{2}\right], \ldots,\left[\mathrm{Z}_{\max (q ; 2 p)}\right],\left[\mathrm{D}_{2}\right], \ldots,\left[\mathrm{D}_{2 q}\right],\left[\Gamma_{T}(2 p) \cup \Gamma_{T}(2 q)\right],\right. \\
&\left.\quad\left[\Gamma_{O}(2 p) \cup \Gamma_{O}(2 q)\right],\left[\Gamma_{I}(2 p) \cup \Gamma_{I}(2 q)\right],[\mathrm{O}(2)],[\mathrm{SO}(3)]\right\} .
\end{aligned}
$$

Proof. Because $[\mathrm{SO}(3)] \in \mathfrak{I}^{k_{i}}(i=1,2)$, it is clear that we will have all $\left[\mathrm{D}_{l}\right]$ for $2 \leq l \leq 2 q$. Then we will have all $\left[\mathrm{Z}_{i}\right] \odot[\mathrm{SO}(3)]$, for $1 \leq i \leq p$. We also have $\left[\mathrm{Z}_{j}\right] \in\left[\mathrm{D}_{j}\right] \odot\left[\mathrm{D}_{j}\right]$, for $1 \leq j \leq 2 q$; this shows that

$$
\left\{[\mathbb{1}],\left[\mathrm{Z}_{2}\right], \ldots,\left[\mathrm{Z}_{\max (q ; 2 p)}\right]\right\} \subset \Im \mathfrak{I}[2 p, 2 q] .
$$

Now, we can observe that the clips product of dihedral groups and $[\mathrm{O}(2)]$ does not generate cyclic groups, and Lemma 5.2 shows that no other cases can be generated with exceptional subgroups.

\section{Isotropy classes of constitutive tensors}

The symmetry classes of an even-order tensor space. Let us consider the CTS $\mathbb{T}^{2 n}$. It is known that this space can be decomposed orthogonally into a full symmetric space and a complementary one which is isomorphic to a tensor space of order $2 n-1$ [Jerphagnon et al. 1978]:

$$
\mathbb{T}^{2 n}=\mathbb{S}^{2 n} \oplus \mathbb{C}^{2 n-1}
$$


Let us consider the $\mathrm{SO}(3)$-isotypic decomposition of $\mathbb{T}^{2 n}$ :

$$
\mathbb{T}^{2 n}=\bigoplus_{k=0}^{2 n} \alpha_{k} \mathbb{H}^{k}, \text { with } \alpha_{2 n}=1
$$

The part related to $\mathbb{S}^{2 n}$ solely contains even-order harmonic tensors with multiplicity one [Jerphagnon et al. 1978], that is,

$$
\mathbb{S}^{2 n}=\bigoplus_{k=0}^{n} \mathbb{M}^{2 k} \text { and } \mathbb{C}^{2 n-1}=\bigoplus_{k=0}^{2 n-1} \alpha_{k}^{\prime} \mathbb{M}^{k} \text { with } \alpha_{k}^{\prime}=\left\{\begin{array}{l}
\alpha_{k} \text { for } k \text { odd } \\
\alpha_{k}-1 \text { for } k \text { even }
\end{array}\right.
$$

Using the clips operator, the symmetry classes of $\mathbb{T}^{2 n}$ can be expressed:

$$
\mathfrak{I}\left(\mathbb{T}^{2 n}\right):=\mathfrak{I}\left(\mathbb{S}^{2 n}\right) \odot \mathfrak{I}\left(\mathbb{C}^{2 n-1}\right) .
$$

Let us first determine the symmetry classes of $\mathbb{S}^{2 n}$. Using the results of the previous section, we have:

\section{Lemma 6.1.}

$$
\begin{aligned}
\Im\left(\mathbb{S}^{2}\right) & =\left\{\left[\mathrm{D}_{2}\right],[\mathrm{O}(2)],[\mathrm{SO}(3)]\right\} \\
\mathfrak{I}\left(\mathbb{S}^{4}\right) & =\left\{[\mathbb{1}],\left[\mathrm{Z}_{2}\right],\left[\mathrm{D}_{2}\right],\left[\mathrm{D}_{3}\right],\left[\mathrm{D}_{4}\right],[\mathrm{O}(2)],[\mathrm{O}],[\mathrm{SO}(3)]\right\} \\
\mathfrak{I}\left(\mathbb{S}^{2 n}\right) & =\left\{[\mathbb{1}],\left[\mathrm{Z}_{2}\right], \ldots,\left[\mathrm{Z}_{2(n-1)}\right],\left[\mathrm{D}_{2}\right], \ldots,\left[\mathrm{D}_{2 n}\right],[\mathrm{O}(2)],[\mathscr{T}],[\mathrm{O}],[\mathcal{F}],[\mathrm{SO}(3)]\right\} \\
& \text { if } n \geq 3 .
\end{aligned}
$$

In particular,

$$
\# \Im\left(\mathbb{S}^{2}\right)=3, \quad \# \Im\left(\mathbb{S}^{4}\right)=8, \quad \# \Im\left(\mathbb{S}^{2 n}\right)=2(2 n+1) .
$$

Proof. The case $n=1$ is obtained as a direct application of Theorem 5.1 and Proposition 4.4. For $n \geq 2$, let us consider Corollary 5.4 in the case of $k_{1}=2(n-1)$ and $k_{2}=2 n$ :

$$
\begin{aligned}
& \Im(2(n-1), 2 n) \\
& \quad:=\left\{[\mathbb{1}],\left[\mathrm{Z}_{2}\right], \ldots,\left[\mathrm{Z}_{2(n-1)}\right],\left[\mathrm{D}_{2}\right], \ldots,\left[\mathrm{D}_{2 n}\right],\left[\Gamma_{T}(2(n-1)) \cup \Gamma_{T}(2 n)\right],\right. \\
& \left.\quad\left[\Gamma_{O}(2(n-1)) \cup \Gamma_{O}(2 n)\right],\left[\Gamma_{I}(2(n-1)) \cup \Gamma_{I}(2 n)\right],[\mathrm{O}(2)],[\mathrm{SO}(3)]\right\} .
\end{aligned}
$$

In the collection of planar isotropy classes, $\left[\mathrm{Z}_{2 n-1}\right]$ and $\left[\mathrm{Z}_{2 n}\right]$ are missing. It should be observed that the clips product $\mathfrak{I}[2(n-1), 2 n] \odot \mathfrak{I}^{2(n-2)}$ can never complete the sequence.

For exceptional groups it can be observed that for any $n \geq 3$ the $\mathrm{SO}(3)$-irreducible decomposition will contain $\mathbb{W}^{6}$. As $\{[\mathscr{T}],[\mathcal{O}],[\mathscr{G}]\}$ are isotropy classes for $\mathbb{T}^{6}$, it would be the same for any space that contains $\mathbb{W}^{6}$.

Therefore, for $n \geq 3$, 
$\Im\left(\mathbb{S}^{2 n}\right)=\left\{[\mathbb{1}],\left[\mathrm{Z}_{2}\right], \ldots,\left[\mathrm{Z}_{2(n-1)}\right],\left[\mathrm{D}_{2}\right], \ldots,\left[\mathrm{D}_{2 n}\right],[\mathrm{O}(2)],[\mathscr{T}],[\mathrm{O}],[\mathscr{F}],[\mathrm{SO}(3)]\right\}$ and \#I $\left(\mathbb{S}^{2 n}\right)=2(2 n+1)$.

For the case $n=2$, we obtain the same result but without the classes $\mathscr{T}$ and $\mathscr{I}$ and, in such a case, $\# \Im\left(\mathbb{S}^{4}\right)=8$.

Definition 6.2. For a given $\mathrm{SO}(3)$ representation on the tensor space $\mathbb{T}^{2 n}(n \geq 3)$, we define

$\mathscr{b}(2 n)$

$=\left\{[\mathbb{1}],\left[\mathrm{Z}_{2}\right], \ldots,\left[\mathrm{Z}_{2 n}\right],\left[\mathrm{D}_{2}\right], \ldots,\left[\mathrm{D}_{2 n}\right],[\mathrm{SO}(2)],[\mathrm{O}(2)],[\mathcal{T}],[\mathrm{O}],[\mathscr{G}],[\mathrm{SO}(3)]\right\}$.

We also define

$$
\begin{aligned}
\mathscr{C}(2) & =\left\{[\mathbb{1}],\left[\mathrm{Z}_{2}\right],\left[\mathrm{D}_{2}\right],[\mathrm{SO}(2)],[\mathrm{O}(2)],[\mathrm{SO}(3)]\right\} \\
\mathscr{C}(4) & =\left\{[\mathbb{1}],\left[\mathrm{Z}_{2}\right], \ldots,\left[\mathrm{Z}_{4}\right],\left[\mathrm{D}_{2}\right], \ldots,\left[\mathrm{D}_{4}\right],[\mathrm{SO}(2)],[\mathrm{O}(2)],[\mathscr{T}],[\mathrm{O}],[\mathrm{SO}(3)]\right\}
\end{aligned}
$$

One can observe that these sets are in fact all the isotropy classes allowed by Hermann's theorem, and we clearly have

$$
\# \mathscr{C}(2)=6, \quad \# \mathscr{C}(4)=12, \quad \# \mathscr{C}(2 n, n \geq 3)=4 n+5 .
$$

Definition 6.3. Let $\mathbb{T}^{2 n}$ be a tensor space which $\mathrm{SO}(3)$-irreducible decomposition is $\mathbb{T}^{2 n} \simeq \bigoplus_{k=0}^{2 n} \alpha_{k} \mathbb{T}^{k}$. $\mathbb{T}^{2 n}$ is said to be even-harmonic (EH) if $\alpha_{2 p+1}=0$ for each $0 \leq p \leq(n-1)$.

Lemma 6.4. The vector space $\mathbb{G}^{2 n}$ of $2 n$-th order tensors with no index symmetries is not $\mathrm{EH}$.

Proof. For $n \geq 1$, the induced reducible $\mathrm{SO}(3)$-representation on $\mathbb{G}^{2 n}=\bigotimes^{2 n} \mathbb{R}^{3}$ is constructed by tensorial products of the vectorial one. Such a construction implies odd-order tensors in the harmonic decomposition of $\mathbb{G}^{2 n}$.

Now we can prove Theorem I, which we restate in the following form:

Theorem I. Let $\mathbb{T}^{2 n}$ be a tensor space, with $n \geq 3$. If $\mathbb{T}^{2 n}$ is EH then $\Im\left(\mathbb{T}^{2 n}\right)=$ $\Im\left(\mathbb{S}^{2 n}\right)$; otherwise, $\mathfrak{I}\left(\mathbb{T}^{2 n}\right)=\mathfrak{I}\left(\mathbb{G}^{2 n}\right)$.

Proof. We consider the $\mathrm{SO}(3)$-irreducible decomposition of $\mathbb{T}^{2 n}$, which can be written $\mathbb{T}^{2 n} \simeq \mathbb{S}^{2 n} \oplus \mathbb{C}^{2 n-1}$. The following inclusions always hold:

$$
\Im\left(\mathbb{S}^{2 n}\right) \subseteq \Im\left(\mathbb{T}^{2 n}\right) \subseteq \Im\left(\mathbb{G}^{2 n}\right) \subseteq \mathscr{C}(2 n) .
$$

If $\mathbb{T}^{2 n}$ is not $\mathrm{EH}$, there exists at least one $k \in \mathbb{N}$ such that $\alpha_{2 k+1} \neq 0$; then

$$
\mathfrak{I}\left(\mathbb{S}^{2 n}\right) \odot[\mathrm{SO}(2)] \subseteq \Im\left(\mathbb{S}^{2 n}\right) \odot \mathfrak{I}\left(\mathbb{C}^{2 n-1}\right)=\mathfrak{I}\left(\mathbb{T}^{2 n}\right),
$$

since, from Theorem 5.1, any odd-order harmonic tensor admits [SO(2)] as a symmetry class. From Lemma 6.1, dihedral groups are contained up to $2 n$ in $\mathfrak{I}\left(\mathbb{S}^{2 n}\right)$, 
so the missing cyclic groups of $\mathfrak{I}\left(\mathbb{S}^{2 n}\right)$ are obtained by clips products with [SO(2)]. Therefore

$$
\Im\left(\mathbb{S}^{2 n}\right) \odot \mathrm{SO}(2)=\mathscr{C}(2 n) ;
$$

hence $\mathfrak{I}\left(\mathbb{T}^{2 n}\right)=\mathscr{C}(2 n)$. Since $\mathbb{G}^{2 n}$ is not EH, $\mathfrak{I}\left(\mathbb{G}^{2 n}\right)=\mathscr{C}(2 n)$. We conclude, as desired, that if $\mathbb{T}^{2 n}$ is not EH then $\mathfrak{I}\left(\mathbb{T}^{2 n}\right)=\mathfrak{I}\left(\mathbb{G}^{2 n}\right)$.

Conversely, if $\mathbb{T}^{2 n}$ is $\mathrm{EH}, \mathbb{C}^{2 n-1}$ contains only even-order irreducible spaces and its leading harmonic spaces are, at most, of order $2(n-1)$. If the orders of the leading harmonic spaces are strictly less than $2(n-1)$, the same analysis as for Lemma 6.1 leads to the same conclusion. Now suppose that $\alpha_{2(n-1)}^{\prime} \geq 1$; using all the previous results, we have

$$
\begin{aligned}
\mathfrak{I}\left(\mathbb{T}^{2 n}\right) & =\mathfrak{I}\left(\mathbb{S}^{2 n}\right) \odot \Im\left(\mathbb{C}^{2 n-1}\right) \\
& =\left(\mathfrak{I}^{2 n} \odot \mathfrak{I}^{2(n-1)}\right) \odot\left(\mathfrak{I}\left(2(n-1), \alpha_{2(n-1)}^{\prime}\right) \odot \Im\left(2(n-2), \alpha_{2(n-2)}^{\prime}\right)\right) \\
& =\left(\mathfrak{I}^{2 n} \odot \mathfrak{I}(2(n-1), 2)\right)=\mathfrak{I}\left(\mathbb{S}^{2 n}\right) \odot \mathfrak{I}^{2(n-1)} .
\end{aligned}
$$

Since $\mathfrak{I}^{2(n-1)}$ does not contain [SO(2)], the missing classes cannot be generated; therefore $\mathfrak{I}\left(\mathbb{T}^{2 n}\right)=\mathfrak{I}\left(\mathbb{S}^{2 n}\right)$.

We must conclude, then, that for any $2 n$-order tensor space, the symmetry classes are the same as those of either $\mathbb{S}^{2 n}$ or $\mathbb{G}^{2 n}$. In the next subsection we investigate under what conditions each of the possibilities holds.

Construction of a CTS. This last subsection will be devoted to the proof of our main result. The space of constitutive tensors is a subspace of linear maps from $\mathbb{E}_{1}$ to $\mathbb{E}_{2}$. As seen in Section 2,

$$
\mathscr{L}\left(\mathbb{E}_{1}, \mathbb{E}_{2}\right) \simeq \mathbb{E}_{1} \otimes \mathbb{E}_{2} \subset \mathbb{T}^{p} \otimes \mathbb{T}^{q} \simeq \mathbb{T}^{2 n=p+q} .
$$

These vector spaces describe the physical quantities involved in the problem under study. We know, from the previous section, that any CTS has as many symmetry classes as either the complete symmetric tensor space, or the generic tensor space. Here we are interested in obtaining the conditions both on $\mathbb{E}_{1}$ to $\mathbb{E}_{2}$ and on the tensor product (symmetric or not) under which $\mathbb{T}^{2 n}$ is even-harmonic, and therefore has a minimal number of symmetry classes. Distinction will be made between coupling and proper tensor spaces, in the sense previously defined in Section 2.

Coupling tensor spaces. We consider here two STS given by their $\mathrm{SO}(3)$ isotypic decompositions:

$$
\mathbb{E}_{1}=\mathbb{T}^{p}=\bigoplus_{i=0}^{p} \beta_{i} \mathbb{M}^{i} \quad \text { and } \quad \mathbb{E}_{2}=\mathbb{T}^{q}=\bigoplus_{i=0}^{q} \gamma_{j} \mathbb{M}^{j},
$$

with $\beta_{p}=\gamma_{q}=1$.

Lemma 6.5. If $\mathbb{E}_{1} \neq \mathbb{E}_{2}, p>q$ and if $\mathbb{T}^{p} \otimes \mathbb{\mathbb { T }}^{q}$ is $E H$, then $\mathbb{T}^{p}$ is $E H$ and $\mathbb{T}^{q}=\mathbb{T}^{0}$. 
Proof. It is sufficient to consider the tensor product of the two leading irreducible spaces, and to use the Clebsch-Gordan product for SO(3) [Jerphagnon et al. 1978; Auffray 2008]. We obtain

$$
\mathbb{M}^{p} \otimes \mathbb{M}^{q}=\bigoplus_{i=|p-q|}^{p+q} \mathbb{\boxplus}^{i}
$$

Therefore $p$ must be even, and $q=0$. Therefore $\mathbb{T}^{q}=\gamma_{0} \mathbb{\boxplus}^{0}$, and by hypothesis $\gamma_{0}=1$. Thus $\mathbb{T}^{p}$ has to be $\mathrm{EH}$.

Lemma 6.6. If $\mathbb{E}_{1}=\mathbb{E}_{2}$ (and then $p=q$ ) and if $\mathbb{T}^{p} \otimes \mathbb{T}^{p}$ is $E H$ then $\beta_{i}=\gamma_{i}$. Furthermore, if $\mathscr{L}\left(\mathbb{E}_{1}\right)$ is not self-adjoint then $\mathbb{T}^{p}=\mathbb{U}^{0}$.

Proof. The demonstration is the same as the preceding proof.

As a direct application of the two preceding two lemmas, we have:

Theorem II. Consider $\mathbb{T}^{2 p}$, the space of coupling tensors between two physics described by two tensor vector spaces $\mathbb{E}_{1}$ and $\mathbb{E}_{2}$. If these tensor spaces have order at least one, then $\mathfrak{I}\left(\mathbb{T}^{2 n}\right)=\mathfrak{I}\left(\mathbb{G}^{2 n}\right)$.

Proper tensor spaces. In this case we have:

Lemma 6.7. Let $\mathbb{E}=\mathbb{E}_{1}=\mathbb{E}_{2}=\mathbb{T}^{p}$. Assume $\mathbb{T}^{2 p} \subset \mathscr{L}(\mathbb{E})$ is self-adjoint and $E H$. Then:

- If $p=2 m+1$, then $\mathbb{\square}^{2 m+1}=\mathbb{U}^{2 m+1}$.

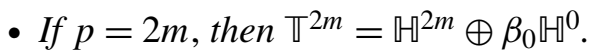

Proof. Because $\mathscr{L}(\mathbb{E})$ is self-adjoint the tensor product is replaced by the symmetric tensor product, and if $\mathbb{T}^{p}=\bigoplus_{i=0}^{p} \beta_{i} \mathbb{M}^{i}$, the symmetric tensor product $\mathbb{T}^{p} \otimes^{S} \mathbb{T}^{p}$ can be decomposed into a direct sum of

$$
\beta_{i}^{2} \mathbb{M}^{i} \otimes \mathbb{S}^{S} \mathbb{\boxplus}^{i} \text { and } \beta_{i} \beta_{j} \mathbb{M}^{i} \otimes \mathbb{M}^{j} \text {, with } i<j \in\{0, \ldots, p\},
$$

with the following Clebsch-Gordan rule for the symmetric product:

$$
\mathbb{M}^{k} \otimes \mathbb{M}^{k}=\bigoplus_{i=0}^{k} \mathbb{H}^{2 i} .
$$

Therefore, we cannot have the tensor product $\mathbb{M}^{i} \otimes \mathbb{W}^{j}$ for $1 \leq i \leq p-1$ and $i \neq j$; thus we deduce that

$$
\mathbb{T}^{p}=\beta_{0} \mathbb{\boxplus}^{0} \oplus \mathbb{\boxplus}^{p} \text { and } \mathbb{T}^{p} \otimes^{S} \mathbb{T}^{p}=\beta_{0} \mathbb{\boxplus}^{0} \oplus\left(\mathbb{\boxplus}^{p} \otimes^{S} \mathbb{\boxplus}^{p}\right) \oplus 2 \beta_{0} \mathbb{\boxplus}^{p} .
$$

Then either $p$ is odd, and $\beta_{0}=0$, or $p$ is even, and $\mathbb{T}^{p}=\beta_{0} \mathbb{H}^{0} \oplus \mathbb{M}^{p}$.

We therefore obtain: 
Theorem III. Consider $\mathbb{T}^{2 p}$, the space of tensors of a proper physics described by the tensor vector space $\mathbb{E}$. If this tensor space is of order $p \geq 3$, and is solely defined in terms of its index symmetries, then $\mathfrak{I}\left(\mathbb{T}^{2 n}\right)=\mathfrak{I}\left(\mathbb{G}^{2 n}\right)$.

Proof. Any tensor subspace defined in terms of its index symmetries contains, as a subspace, the space of full symmetric tensors. Since $p=3$, the harmonic decomposition of $\mathbb{S}^{3}$ does not satisfy the condition of Lemma 6.7. Direct application of this lemma leads to the conclusion.

Remark 6.8. It can be observed that CTS having a minimum number of classes can nevertheless be constructed. They consist in spaces of self-adjoint linear applications between harmonic spaces, which are defined both from complete symmetry under index permutations and a traceless property.

\section{Conclusion}

In this paper the symmetry class determination of even-order tensors has been studied. Based on a new geometric approach, a complete and general answer to this recurrent problem in continuum mechanics has been given. Application of our results solves problems directly that would have been difficult to manage with the Forte-Vianello method. As an example, and for the first time, the symmetry classes of the even-order tensors involved in Mindlin second strain-gradient elasticity were given. To reach this goal a geometric tool, called the clips operator, has been introduced. Its main properties and all the products for $\mathrm{SO}(3)$-closed-subgroups were also provided. We believe that these results may find applications in other contexts. Using the geometrical framework introduced in this paper, some extensions of the current method can be considered:

- Extending this approach to odd-order tensors.

- Taking into account the coexistence of different symmetry properties for the physical properties of architectured multimaterials.

These extensions will be the objects of forthcoming papers.

\section{Appendix: Clips operation on SO(3)-subgroups}

Here we establish results concerning the clips operator on $\mathrm{SO}(3)$-subgroups. The geometric idea to study the intersection of symmetry classes relies on the symmetry determination of composite figures the symmetry groups of which are the intersection of two elementary figures. As an example we consider the rotation $\mathbf{r}=\mathbf{Q}(\mathbf{k} ; \pi / 3)$; determining $\mathrm{D}_{4} \cap \mathbf{r} \mathrm{D}_{4} \mathbf{r}^{t}$ is tantamount to establishing the set of transformations letting the composite Figure 1 invariant. 


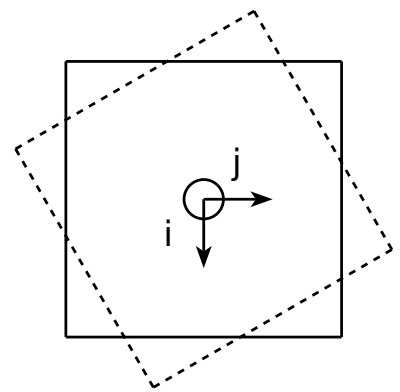

Figure 1. Composite figure associated with $\mathrm{D}_{4} \cap \mathbf{r D}_{4} \mathbf{r}^{t}$, where $\mathbf{r}=\mathbf{Q}(\mathbf{k} ; \pi / 3)$.

Parametrization of subgroups. We will define geometric elements for each $\mathrm{SO}(3)$ closed subgroup:

- The cyclic group $\mathrm{Z}_{n}$ is characterized by the $O z$ axis; it will be denoted by $\mathrm{Z}_{n}^{0}:=\mathrm{Z}_{n}$.

- The same convention is retained for the dihedral group $\mathrm{D}_{n}$, that is, $\mathrm{D}_{n}^{0}:=\mathrm{D}_{n}$.

- For the cube $\mathscr{C}_{0}$ (see Figure $2 \mathrm{a}$ ) we defined its vertex collection $\left\{A_{i}\right\}_{i=1 \ldots 8}=$ $( \pm 1 ; \pm 1 \pm 1) ; \mathscr{C}_{0}$ is $\mathbb{O}^{0}$-invariant.

- For the tetrahedron we consider Figure $2 \mathrm{a}$ and define $\mathscr{T}_{0}$ to be the tetrahedron $A_{1} A_{3} A_{7} A_{5} ; \mathscr{T}_{0}$ is $\mathscr{T}^{0}$-invariant.

- For the dodecahedron (see Figure $2 b$ ), we denote by $\mathscr{D}_{0}$ the figure with the following vertices (where $\phi$ is the golden ratio):

- twelve vertices of type $\left( \pm a / 2, \pm \phi^{2} a / 2,0\right)$ circularly permuted and

- eight vertices of a cube with coordinates $( \pm \phi a / 2, \pm \phi a / 2, \pm \phi a / 2)$.

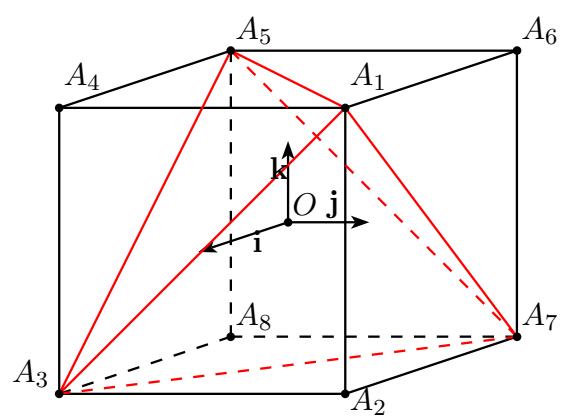

(a)

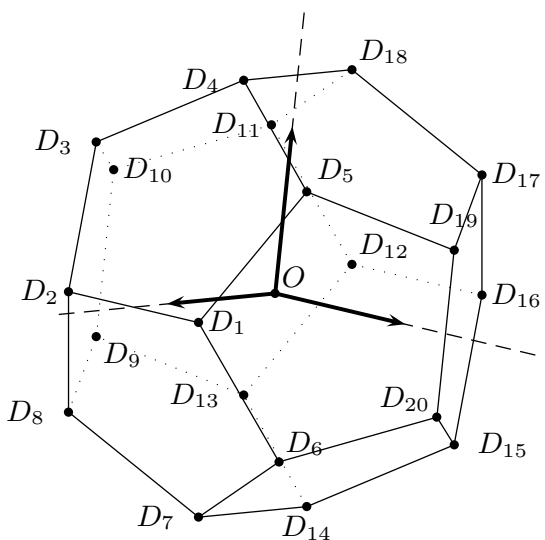

(b)

Figure 2. Cube $\mathscr{C}_{0}$ (a) and dodecahedron $\mathscr{D}_{0}(\mathrm{~b})$. 
Axes and subgroup classes. For every $\mathrm{SO}(3)$-subgroup, we defined its $g$ conjugate in the following way: $K^{g}=g K^{0} g^{t}$, where the superscript $g$ indicates the transformation, and 0 the initial configuration. To proceed towards our analysis we need to introduce the following group decomposition [Ihrig and Golubitsky 1984; Golubitsky et al. 1988].

Definition A.1. Let $K_{1}, K_{2}, \ldots, K_{s}$ be subgroups of $\Sigma$. Then $\Sigma$ is the direct union of the $K_{i}$ if

$$
\Sigma=\bigcup_{i=1}^{s} K_{i} \quad \text { and } \quad K_{i} \cap K_{j}=\{e\} \text { if } i \neq j .
$$

In this case we write $K=\biguplus_{i=1}^{s} K_{i}$.

We give some important details about the geometric structure of an $\mathrm{SO}(3)$ subgroup:

- $\mathrm{Z}_{n}^{0}$ is characterized by the $O z$ axis, generated by $\mathbf{k}$. For every rotation $g \in$ $\mathrm{SO}(3)$, we denote by $a$ the axis generated by $g \mathbf{k}$ and let $Z_{n}^{a}=Z_{n}^{g}$ indicate the rotation axis.

- $\mathrm{D}_{n}^{0}$ is characterized by its primary axis $O z$ and several secondary axes $b_{l}$. Therefore

$$
\mathrm{D}_{n}^{0}=\mathrm{Z}_{n}^{0} \biguplus_{l=0}^{n-1} \mathrm{Z}_{2}^{b_{l}}
$$

Each $b_{l}$ is perpendicular to $O z$. They are related by the $Z_{n}^{0}$ generator. $\mathrm{D}_{n}^{0}$ is chosen such that one $b_{l}$ is generated by $\mathbf{i}$. For every rotation $g \in \mathrm{SO}(3)$ we define $a$ - generated by $g \mathbf{k}$ - to be the primary axis and $b-$ generated by $g \mathbf{i}$ - to be the secondary one; this is denoted by

$$
\mathrm{D}_{n}^{a, b}=\mathrm{D}_{n}^{g}
$$

- The subgroup $\mathscr{T}^{0}$ can be split into a direct union of cyclic subgroups [Ihrig and Golubitsky 1984]:

$$
\mathscr{T}^{0}=\biguplus_{i=1}^{4} \mathrm{Z}_{3}^{v t_{i}} \uplus \biguplus_{j=1}^{3} \mathrm{Z}_{2}^{e t_{j}},
$$

where the vertex axes of the tetrahedron are written $v t_{i}$ and the edge axes $e t_{j}$; the details of these axes appear in Figure 2a. Each conjugate subgroup $\mathscr{T}^{g}$ will be characterized by the set of its axes $\left(g v t_{i}, g e t_{i}\right), g \in \mathrm{SO}(3)$.

- The octahedral subgroup $0^{0}$ splits into

$$
\mathrm{O}^{0}=\biguplus_{i=1}^{3} \mathrm{Z}_{4}^{f c_{i}} \uplus \biguplus_{j=1}^{4} \mathrm{Z}_{3}^{v c_{j}} \uplus \biguplus_{l=1}^{6} \mathrm{Z}_{2}^{e c_{l}},
$$




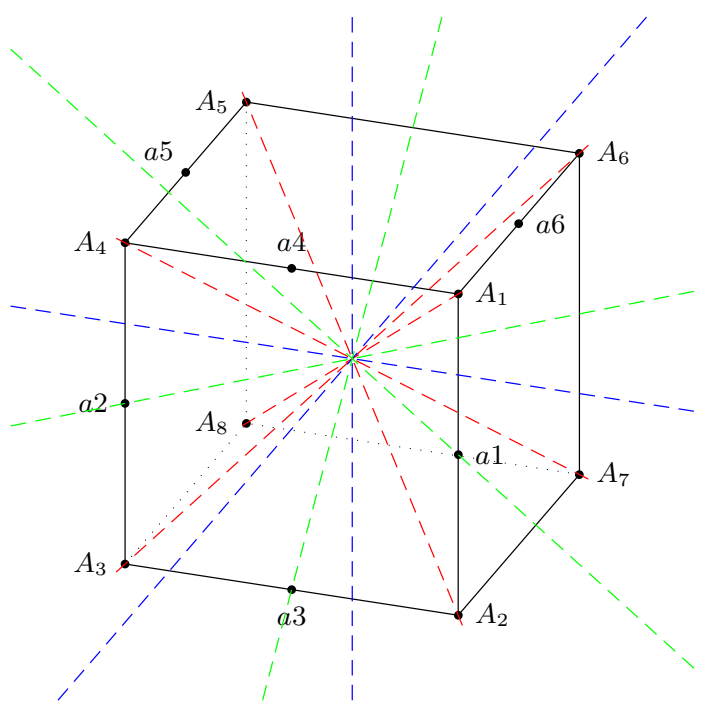

Figure 3. Symmetry axes of $\mathscr{C}_{0}$.

where the vertex, edge, and face axes are denoted respectively by $v c_{i}, e c_{j}$, and $f c_{j}$. Details can be found in Figure 3. For every rotation $g \in \mathrm{SO}(3), \mathbb{O}^{g}$ is characterized by its set of transformed axes $\left(g f c_{i}, g e c_{j}, g v c_{l}\right)$.

- The icosahedral group $\mathscr{\Phi}^{0}$ splits into

$$
g^{0}=\biguplus_{i=1}^{6} \mathrm{Z}_{5}^{f d_{i}} \uplus \biguplus_{j=1}^{10} \mathrm{Z}_{3}^{v d_{j}} \uplus \biguplus_{l=1}^{15} \mathrm{Z}_{2}^{e d_{l}},
$$

where the vertex, edge, and face axes are denoted respectively by $v d_{i}, e d_{j}$, and $f d_{j}$; the details can be found in Figure $2 \mathrm{~b}$. The vertex axes $v d_{j}$ are characterized by the vertices $D_{j}$ for $j=1 \ldots 10$.

\section{Planar subgroups.}

Cyclic subgroups. We begin with the following lemma.

Lemma A.2. For every two integers $m$ and $n$ greater than 2, and for every two axes $a$ and $b$ :

- If $a \neq b$ then $\mathrm{Z}_{n}^{a} \cap \mathrm{Z}_{m}^{b}=\mathbb{1}$.

- If $a=b$ then by setting $d:=\operatorname{gcd}(m, n)$ we will have $\mathrm{Z}_{n}^{a} \cap \mathrm{Z}_{m}^{b}=\mathrm{Z}_{d}^{a}$.

Proof. Let $g \in \mathrm{Z}_{n}^{a} \cap \mathrm{Z}_{m}^{b}$, with $a \neq b$. Both $a$ and $b$ are generated by two noncollinear eigenvectors for $g$, with eigenvalue 1 . As $\operatorname{det} g=1$ the third eigenvalue is also 1 , therefore $g=e$. Thus we have the first point of the lemma. If now we take, for example, a common rotation of $Z_{n}^{0}$ and $Z_{m}^{0}$, then this rotation corresponds to an 
angle $\theta=2 l \pi / n=2 r \pi / m$ with $r$ and $l$ integers. Thus $l m=r n$ and, setting $m=d m_{1}$ and $n=d n_{1}$, we will have $l m_{1}=r n_{1}$. As $m_{1}$ and $n_{1}$ are relatively prime, we deduce that

$$
l=\alpha n_{1} \text { and then } \theta=\frac{2 l \pi}{n}=\frac{2 \alpha \pi}{d} \in \mathrm{Z}_{d}^{0} .
$$

The converse inclusion is obvious, so we can conclude the lemma.

A direct application of Lemma A.2 to the intersection $Z_{n}^{0} \cap Z_{n}^{g}$ leads to the result:

Lemma A.3. For all integers $n$ and $m$, we set $d=\operatorname{gcd}(n, m) ;$ we have $\left[\mathrm{Z}_{n}\right] \odot\left[\mathrm{Z}_{m}\right]=$ $\left\{[\mathbb{1}],\left[\mathrm{Z}_{d}\right]\right\}$.

Dihedral subgroups. Let us consider first the intersection $\Gamma=\mathrm{D}_{n}^{0} \cap \mathrm{Z}_{m}^{a}$. As $\mathrm{D}_{n}^{0}=$ $\mathrm{Z}_{n}^{0} \biguplus_{l=1}^{n} \mathrm{Z}_{2}^{b_{l}}$ the following cases have to be considered:

- When $O z=a$, the intersection $\Gamma=\mathrm{Z}_{n}^{0} \cap \mathrm{Z}_{m}^{a}$ and one can apply Lemma A.2.

- When, for some $l$, we have $a=b_{l}$, then $Z_{n}^{0} \cap Z_{m}^{a}=\mathbb{1}$ and one has to consider $\mathrm{Z}_{2}^{b} \cap \mathrm{Z}_{m}^{a}$, which equals the identity as soon as $m$ is odd.

Lemma A.4. For every two integers $n$ and $m$, we set $d:=\operatorname{gcd}(n, m)$ and $d_{2}(m):=$ $\operatorname{gcd}(m, 2)$; then we have

$$
\left[\mathrm{D}_{n}\right] \odot\left[\mathrm{Z}_{m}\right]=\left\{[\mathbb{1}],\left[\mathrm{Z}_{d_{2}(m)}\right],\left[\mathrm{Z}_{d}\right]\right\} .
$$

Now consider the second kind of intersection:

$$
\Gamma=\mathrm{D}_{n}^{0} \cap \mathrm{D}_{m}^{g}=\left(\mathrm{D}_{n}^{0}=\mathrm{Z}_{n}^{0} \uplus \biguplus_{l=1}^{n} \mathrm{Z}_{2}^{b_{l}}\right) \cap\left(\mathrm{D}_{m}^{g}=\mathrm{Z}_{m}^{a} \uplus \biguplus_{l=1}^{m} \mathrm{Z}_{2}^{g b_{l}}\right) .
$$

The following cases have to be considered:

- When $O z=a$ and $O x=g b_{l} 0$ for some $l$ : if $d=1$ then $\Gamma=Z_{2}^{b_{0}}$, otherwise $\Gamma=\mathrm{D}_{m}^{0}$.

- When $O z=a$ and $O x \neq g b_{l}: \Gamma=Z_{d}^{0}$.

- When $O z=g b_{l}$ for some $l$ : if $n$ is even then $\Gamma=\mathrm{Z}_{2}$, otherwise $\Gamma=\mathbb{1}$. The results are the same when the primary axis of $\mathrm{D}_{m}^{g}$ coincides with a secondary axis of $\mathrm{D}_{n}^{0}$.

Lemma A.5. For all integers $n$ and $m$, we set $d:=\operatorname{gcd}(n, m)$ and

$$
d_{2}(m):=\operatorname{gcd}(m, 2), \quad d_{2}(n):=\operatorname{gcd}(n, 2), \quad d z:=\left\{\begin{array}{l}
2 \text { if } d=1, \\
1 \text { otherwise } .
\end{array}\right.
$$

Then we have $\left[\mathrm{D}_{n}\right] \odot\left[\mathrm{D}_{m}\right]=\left\{[\mathbb{1}],\left[\mathrm{Z}_{d_{2}(n)}\right],\left[\mathrm{Z}_{d_{2}(m)}\right],\left[\mathrm{Z}_{d z}\right],\left[\mathrm{Z}_{d}\right],\left[\mathrm{D}_{d}\right]\right.$. 
Clips operations on exceptional and maximum subgroups. Here we are concerned with the subgroups $\mathscr{T}^{0}, \mathrm{O}^{0}, g^{0}, \mathrm{SO}(2)^{0}$, and $\mathrm{O}(2)^{0}$. For these studies, we will use results concerning their proper subgroups [Ihrig and Golubitsky 1984]. This information is summed up in the following diagram [Auffray et al. 2011], whose arrows are to be understood as inclusion of conjugates.

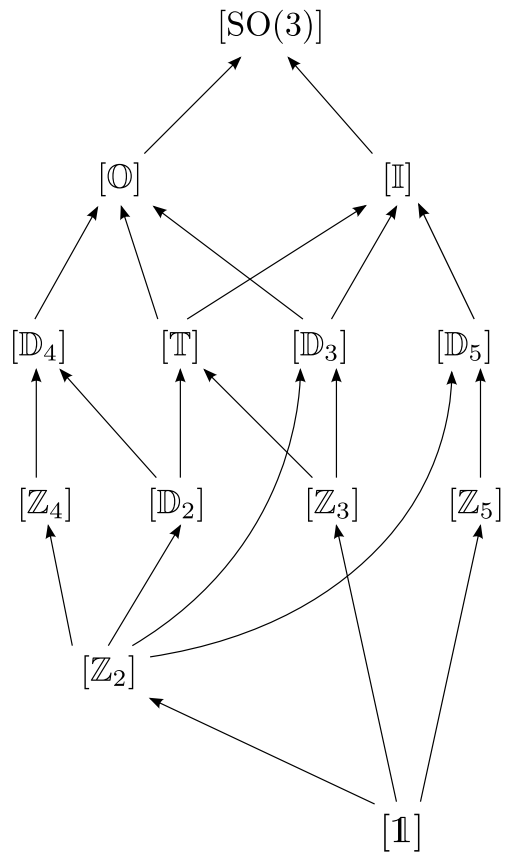

Figure 4. Exceptional subgroups in the poset of the closed subgroup of $\mathrm{SO}(3)$.

Tetrahedral subgroup. We revisit the decomposition (A.2):

$$
\mathscr{T}^{0}=\mathrm{Z}_{3}^{v t_{1}} \uplus \mathrm{Z}_{3}^{v t_{2}} \uplus \mathrm{Z}_{3}^{v t_{3}} \uplus \mathrm{Z}_{3}^{v t_{4}} \uplus \mathrm{Z}_{2}^{e t_{1}} \uplus \mathrm{Z}_{2}^{e t_{2}} \uplus \mathrm{Z}_{2}^{e t_{3}} .
$$

We begin by studying $\mathscr{T}^{0} \cap \mathrm{Z}_{n}^{a}$. As a consequence of Lemma A.2, the primary axis of $Z_{n}^{a}$ must be an edge axis or a face axis of the tetrahedron. We therefore obtain:

Lemma A.6. For every integer $n$, we set $d_{2}(n):=\operatorname{gcd}(n, 2)$ and $d_{3}(n):=\operatorname{gcd}(3, n)$; then we have $\left[\mathrm{Z}_{n}\right] \odot[\mathscr{T}]=\left\{[\mathbb{1}],\left[\mathrm{Z}_{d_{2}(n)}\right],\left[\mathrm{Z}_{d_{3}(n)}\right]\right\}$.

Now let us consider $\Gamma=\mathscr{T}^{0} \cap \mathrm{D}_{n}^{g}$. We will use the primary and secondary axes of the dihedral subgroup:

$$
\mathrm{D}_{n}^{g}=\mathrm{Z}_{n}^{a} \biguplus \mathrm{Z}_{2}^{b}
$$

We recall that the vertex axes of the tetrahedron are denoted by $v t_{i}$ and the edge axes are denoted by $e t_{j}$. It is clear that $\Gamma$ is a subgroup of $\mathscr{T}^{0}$. Furthermore: 
- As soon as $3 \mid n$ if $a=v t_{i}$ then $\Gamma=Z_{3}$ is maximal.

- When $2 \mid n$ we can find $g$ such that $a=e t_{j}$. Then if $b=e t_{j}, \Gamma=\mathrm{D}_{2}$; otherwise $\Gamma=\mathrm{Z}_{2}$.

- In any case, when we only have $b=e t_{j}$, then $\Gamma=Z_{2}$.

Finally we can deduce the lemma:

Lemma A.7. For every integer $n$ we denote $d_{2}(n):=\operatorname{gcd}(2, n)$ and $d_{3}(n):=$ $\operatorname{gcd}(3, n)$; then we have $\left[\mathrm{D}_{n}\right] \odot[\mathcal{T}]=\left\{[\mathbb{1}],\left[\mathrm{Z}_{2}\right],\left[\mathrm{Z}_{d_{3}(n)}\right],\left[\mathrm{D}_{d_{2}(n)}\right]\right\}$.

Now, for the study of $\Gamma=\mathscr{T}^{0} \cap \mathscr{T}^{g}$ the arguments will be based on $\mathscr{T}$ subgroups as well as on the axes.

- First, we can find a $g$ such that all the axes are modified; in this case $\Gamma=\mathbb{1}$.

- A rotation around a face or an edge axis can be found such that only this axis is left fixed. Then $\Gamma=Z_{2}$ or $\Gamma=Z_{3}$ depending on the fixed axis.

- If we have $\Gamma \supset \mathrm{D}_{2}$ then we can deduce that $g$ carries two edge axes onto two edge axes. After a given permutation of axes (which leaves fixed $\mathscr{T}^{0}$ ) we can suppose that $g$ leaves fixed axes $v t_{1}$ and $v t_{2}$; we then conclude that $g$ fixes also the axis $v t_{3}$ and then $\mathscr{T}^{g}=\mathscr{T}^{0}$. Thus we have here $\Gamma=\mathscr{T}^{0}$.

We deduce here the following lemma:

Lemma A.8. We have $[\mathscr{T}] \odot[\mathscr{T}]=\left\{[\mathbb{1}],\left[Z_{2}\right],\left[Z_{3}\right],[\mathscr{T}]\right.$.

Octahedral subgroup. We begin by taking back the decomposition (A.3):

$$
0^{0}=\biguplus_{i=1}^{3} \mathrm{Z}_{4}^{f c_{i}} \uplus \biguplus_{j=1}^{4} \mathrm{Z}_{3}^{v c_{j}} \uplus \biguplus_{l=1}^{6} \mathrm{Z}_{2}^{e c_{l}} .
$$

As in the case of the tetrahedron, we directly get the lemma:

Lemma A.9. For every integer $n$, we write

$$
d_{2}(n)=\operatorname{gcd}(n, 2), \quad d_{3}(n)=\operatorname{gcd}(n, 3), \quad d_{4}(n)= \begin{cases}4 & \text { if } 4 \mid n, \\ 1 & \text { otherwise. }\end{cases}
$$

Then we have $\left[\mathrm{Z}_{n}\right] \odot[0]=\left\{[\mathbb{1}],\left[\mathrm{Z}_{d_{2}(n)}\right],\left[\mathrm{Z}_{d_{3}(n)}\right],\left[\mathrm{Z}_{d_{4}(n)}\right]\right.$.

To study the clips operation with dihedral groups, we proceed in the same way as for the tetrahedron subgroup. The purpose is to examine axes of the cube and dihedral group. The arguments are about the same in each case. Therefore we will only detail the cases where $4 \nmid n, 3 \mid n$ and $n$ is odd.

- If $a=v c_{j}$ : then if $b=f c_{j}, \Gamma=\mathrm{D}_{3}$; otherwise $\Gamma=\mathrm{Z}_{3}$.

- If $a=f c_{j}$ : then if $b=f c_{j}, \Gamma=\mathrm{Z}_{2}$; otherwise $\Gamma=\mathbb{1}$.

- If $a=e c_{j}$ : then if $b=e c_{j}$ or $b=f c_{j}, \Gamma=\mathrm{Z}_{2}$. 
All these arguments leads us to the following lemma:

Lemma A.10. For every integer $n$, we set

$$
d_{2}(n):=\operatorname{gcd}(n, 2), \quad d_{3}(n):=\operatorname{gcd}(n, 3), \quad d_{4}(n):= \begin{cases}4 & \text { if } 4 \mid n, \\ 1 & \text { otherwise. }\end{cases}
$$

Then we have $\left[\mathrm{D}_{n}\right] \odot[0]=\left\{[\mathbb{1}],\left[\mathrm{Z}_{2}\right],\left[\mathrm{Z}_{d_{3}(n)}\right],\left[\mathrm{Z}_{d_{4}(n)}\right],\left[\mathrm{D}_{d_{2}(n)}\right],\left[\mathrm{D}_{d_{3}(n)}\right],\left[\mathrm{D}_{d_{4}(n)}\right]\right\}$.

Now, we take $\Gamma=\mathscr{O}^{0} \cap \mathscr{T}^{g}$ and one can observe that if $\Gamma$ is necessarily a common subgroup of $0^{0}$ and $\mathscr{T}^{g}$, then its class must contain (in the sense of the partial order) $[\mathbb{1}],\left[\mathrm{Z}_{2}\right],\left[\mathrm{Z}_{3}\right],\left[\mathrm{D}_{2}\right]$, or $[\mathscr{T}]$. After that:

- There exists a rotation $g$ around an edge axis of $\mathscr{T}^{0}$ (that is, a common face axis of the cube) such that only this axis is fixed; and then $\Gamma=Z_{2}$.

- There exists a rotation $g$ around a vertex axis of $\mathscr{T}^{0}$ (that is, a common vertex axis of the cube) such that only this axis is fixed; and then $\Gamma=Z_{3}$.

- As soon as $\Gamma \supset \mathrm{D}_{2}$, as in the tetrahedral case, we necessarily have $\Gamma=\mathscr{T}^{0}$.

We conclude here the lemma:

Lemma A.11. We have $[\mathscr{T}] \odot[\mathcal{O}]=\left\{[\mathbb{1}],\left[\mathrm{Z}_{2}\right],\left[\mathrm{Z}_{3}\right],[\mathscr{T}]\right\}$.

For the study of $\Gamma=0^{0} \cap \mathbb{O}^{g}$ we will also use arguments based on subgroups. Some results are nevertheless more subtle:

- First, there exists a rotation $g$ that fixes only one edge axis, and in that case $\Gamma=\mathrm{Z}_{2}$.

- Then there exists a rotation that leaves fixed only one vertex axis, and in that case $\Gamma=\mathrm{Z}_{3}$.

- There exists also a rotation that leaves fixed only one face axis, and no other axis is fixed. See the figure below, which illustrates the case $\Gamma=Z_{4}$.

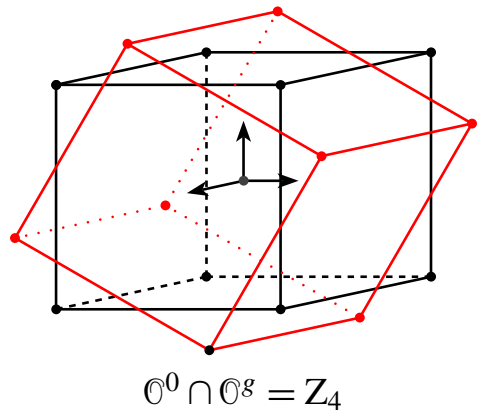

- We can also find a rotation that leaves fixed a face axis and which brings an edge axis onto a face axis. Indeed, when we take $g=\mathbf{Q}(\mathbf{i} ; \pi / 4)$ we obtain $\Gamma=Z_{4}^{\mathbf{i}} \uplus Z_{2}^{\mathbf{k}}=\mathrm{D}_{4}$; see left figure on the next page. 

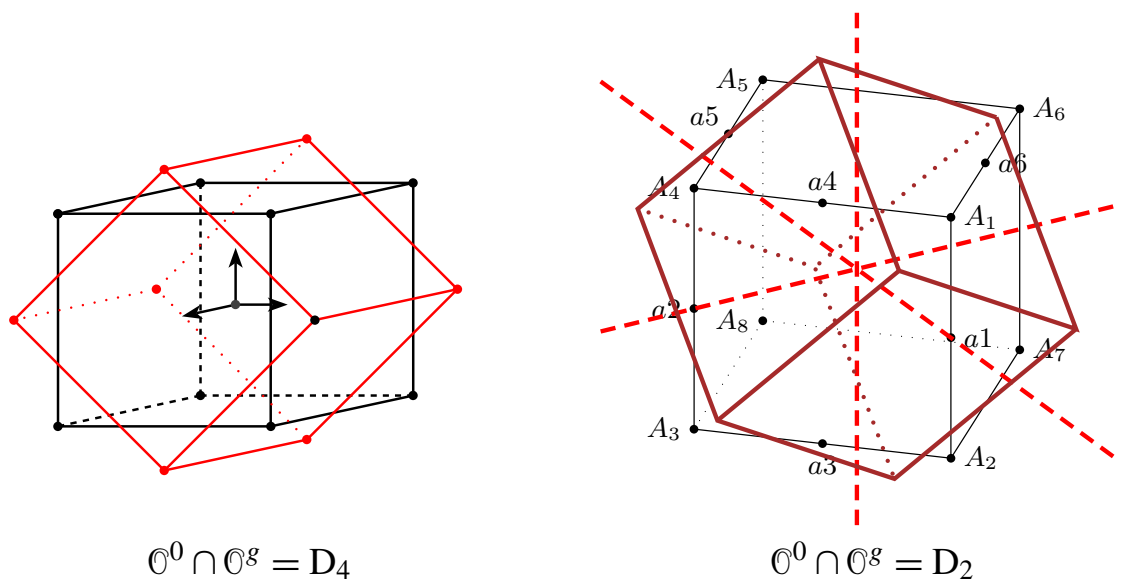

- If we take $g=\mathbf{Q}(\mathbf{k} ; \pi / 4) \circ \mathbf{Q}(\mathbf{i} ; \pi / 4)$ we directly obtain $\Gamma=\mathrm{D}_{2}$. We can exactly compute that $g f c_{3}=e c_{6}, g e c_{1}=f c_{1}$, and $g e c_{2}=e c_{5}$ and no other axes correspond; see figure above and to the right.

- If we take $g=\mathbf{Q}\left(v c_{1}, \pi\right)$ we will find $\Gamma=\mathrm{D}_{3}$ with $v c_{1}$ as primary axis and ec5 a secondary axis.

- If $\Gamma \supset \mathscr{T}$ then, necessarily, $g$ leaves fixed the three edge axes of the tetrahedron, and then $g$ will fix the cube $\mathscr{C}_{0}$; thus $\Gamma=0^{0}$.

Finally we get the lemma:

Lemma A.12. We have $[0] \odot[0]=\left\{[\mathbb{1}],\left[\mathrm{Z}_{2}\right],\left[\mathrm{D}_{2}\right],\left[\mathrm{Z}_{3}\right],\left[\mathrm{D}_{3}\right],\left[\mathrm{Z}_{4}\right],\left[\mathrm{D}_{4}\right],[\mathrm{O}]\right\}$.

Icosahedral subgroup. We take the decomposition (A.4):

$$
g^{0}=\biguplus_{i=1}^{6} \mathrm{Z}_{5}^{f d_{i}} \uplus \biguplus_{j=1}^{10} \mathrm{Z}_{3}^{v d_{j}} \uplus \biguplus_{l=1}^{15} \mathrm{Z}_{2}^{e d_{l}} .
$$

As in the previous situations, we directly get the lemma:

Lemma A.13. For every integer $n$, we set

$$
d_{2}:=\operatorname{gcd}(n, 2), \quad d_{3}:=\operatorname{gcd}(n, 3), \quad d_{5}:=\operatorname{gcd}(n, 5) .
$$

Then we have $\left[\mathrm{Z}_{n}\right] \odot[\mathscr{F}]=\left\{[\mathbb{1}],\left[\mathrm{Z}_{d_{2}}\right],\left[\mathrm{Z}_{d_{3}}\right],\left[\mathrm{Z}_{d_{5}}\right]\right\}$.

Now, for the study of $g^{0} \cap \mathrm{D}_{n}^{g}$ we again use the arguments about axes:

- If $a=f t_{j}$ or $a=v t_{j}$, then $\Gamma \in\left\{Z_{d_{3}}, Z_{d_{5}}, \mathrm{D}_{d_{3}} \mathrm{D}_{d_{5}}\right\}$.

- If $a=e t_{j}$ then $\Gamma \in\left\{Z_{d_{2}}, \mathrm{D}_{d_{2}}\right\}$.

When we argue on the secondary axis of $\mathrm{D}_{n}^{g}$, we see that we can always have $\mathrm{Z}_{2}$. Finally we get the lemma: 
Lemma A.14. For every integer $n$, we set

$$
d_{2}:=\operatorname{gcd}(n, 2), \quad d_{3}:=\operatorname{gcd}(n, 3), \quad d_{5}:=\operatorname{gcd}(n, 5) .
$$

Then we have

$$
\left.\left[\mathrm{D}_{n}\right] \odot[\mathscr{I}]=\left\{[\mathbb{1}],\left[\mathrm{Z}_{2}\right],\left[\mathrm{Z}_{d_{3}}\right],\left[\mathrm{Z}_{d_{5}}\right],\left[\mathrm{D}_{d_{2}}\right],\left[\mathrm{D}_{d_{3}}\right],\left[\mathrm{D}_{d_{5}}\right]\right\}\right\} .
$$

For the intersection $\mathscr{I}^{0} \cap \mathscr{T}^{g}$ it is clear, because of the inclusion $\mathscr{T}^{0} \subset \mathscr{I}^{0}$, that we can obtain all the classes of $[\mathscr{T}] \odot[\mathscr{T}]$. If now this intersection contains a subgroup $\mathrm{D}_{2}$, we will necessarily have

$$
\mathrm{D}_{2}=\mathrm{Z}_{2}^{\text {get }_{1}} \uplus \mathrm{Z}_{2}^{\text {get }_{3}} \uplus \mathrm{Z}_{2}^{\text {get }} \text {, }_{3},
$$

where get $_{i}$ are the three edge axis of the tetrahedron $g \mathscr{T}_{0}$. These three axes will then have to correspond to three perpendicular axes of the dodecahedron. After permutation of the axes, which leaves the dodecahedron fixed, we can suppose that these three axes are generated by the three vectors of the basis. But, then, the vertex axes of the tetrahedron will correspond to vertex axes of the embedded cube in the dodecahedron. We can then deduce that the intersection will be the whole $\mathscr{T}$ subgroup.

Now we have to study $\Gamma=g^{0} \cap \mathcal{O}^{g}$. For that, we refer to the common subgroups of [O] and [F]. Such subgroups can clearly be taken from the poset on page 203. First, it is clear that, when the cube related to $0^{g}$ is the embedded cube in the dodecahedron, we will have $\Gamma=\mathscr{T}$.

We also can find a rotation $g$ such that $\Gamma$ contains $\mathrm{D}_{3}$ : indeed, $g$ has to bring the vertex axis of the cube $v c_{1}$ onto the vertex axis of the dodecahedron $v d_{5}$ and the edge axis of the cube $e c_{5}$ onto the edge axis of the dodecahedron $e d_{7}$. With the maximality argument, we can deduce that $\Gamma=\mathrm{D}_{3}$. We now have to examine the case of $\mathrm{D}_{2}, \mathrm{Z}_{3}$, and $\mathrm{Z}_{2}$ :

- When $\Gamma \supset \mathrm{D}_{2}$, then, after permuting of the axes of the dodecahedron, we can suppose that $g$ leaves fixed the three axes of the basis vector. But these three axes are axes of rotation of order 2 of the dodecahedron. Thus, $g$ will fix the cube $\mathscr{C}_{0}$ and we can deduce that $\mathbb{O}^{g}=\mathcal{O}^{0}$. We then have $\Gamma=\mathscr{T}$.

- We can find a rotation $g$ around a vertex axis, for example, $v d_{5}$ such that $\Gamma=\mathrm{Z}_{3}$.

- As above, we can find a rotation around an edge axis, such that $\Gamma=\mathrm{Z}_{2}$.

We finally conclude with the formula:

$$
[\mathrm{O}] \odot[\mathscr{F}]=\left\{[\mathbb{1}],\left[\mathrm{Z}_{2}\right],\left[\mathrm{Z}_{3}\right],\left[\mathrm{D}_{3}\right],[\mathscr{T}]\right\} .
$$

For the intersection $\Gamma=\mathscr{I}^{0} \cap \mathscr{I}^{g}$ we will have to study the case of classes [ $\left.\mathscr{T}\right],\left[\mathrm{D}_{3}\right]$, $\left[\mathrm{D}_{5}\right],\left[\mathrm{D}_{2}\right],\left[\mathrm{Z}_{3}\right],\left[\mathrm{Z}_{5}\right]$, and $\left[\mathrm{Z}_{2}\right]$ : 
- When $\Gamma \supset \mathscr{T}$ or $\Gamma \supset \mathrm{D}_{2}$, it then contains all the three second-order rotations around each base axis, which will be three edge axes of the dodecahedron. Thus we can deduce that $g$, after permutation of these axes, leaves fixed three perpendicular axes, and then $g$ leaves fixed $\mathscr{l}^{0}$; finally $\Gamma=\mathscr{I}$.

- There exists a rotation $g$ around an edge axis so that $\Gamma=Z_{2}$. The same argument leads us to $Z_{3}$ and $Z_{5}$.

- If we take $g$ to be the second-order rotation around the axis $v d_{3}$, we can compute that this rotation only leaves fixed the axes $v d_{3}, e d_{6}, e d_{8}$, and $e d_{15}$, and then $\Gamma=\mathrm{D}_{3}$.

- If we take $g$ to be the second-order rotation around the face axis $f d_{1}$ we can also compute that it only leaves fixed the axes $f d_{1}, e d_{7}, e d_{11}, e d_{12}$, and $e d_{14}$, thus $\Gamma=\mathrm{D}_{5}$.

\section{References}

[Abud and Sartori 1983] M. Abud and G. Sartori, "The geometry of spontaneous symmetry breaking”, Ann. Phys. 150:2 (1983), 307-372.

[Auffray 2008] N. Auffray, "Décomposition harmonique des tenseurs: méthode spectrale", $C$. $R$. Mécanique 336:4 (2008), 370-375.

[Auffray et al. 2011] N. Auffray, B. Kolev, and M. Petitot, "Invariant-based approach to symmetry class detection”, preprint, 2011. arXiv 1111.0861

[Auffray et al. 2013] N. Auffray, H. Le Quang, and Q.-C. He, "Matrix representations for 3D straingradient elasticity", J. Mech. Phys. Solids (2013).

[Backus 1970] G. Backus, “A geometrical picture of anisotropic elastic tensors”, Rev. Geophys. 8:3 (1970), 633-671.

[Bóna et al. 2004] A. Bóna, I. Bucataru, and M. A. Slawinski, "Characterization of elasticity-tensor symmetries using SU(2)”, J. Elasticity 75:3 (2004), 267-289.

[Bredon 1972] G. E. Bredon, Introduction to compact transformation groups, Pure and Applied Mathematics 46, Academic Press, New York, 1972.

[Chadwick et al. 2001] P. Chadwick, M. Vianello, and S. C. Cowin, "A new proof that the number of linear elastic symmetries is eight”, J. Mech. Phys. Solids 49:11 (2001), 2471-2492.

[dell'Isola et al. 2009] F. dell'Isola, G. Sciarra, and S. Vidoli, “Generalized Hooke's law for isotropic second gradient materials", Proc. R. Soc. Lond. A 465:2107 (2009), 2177-2196.

[dell'Isola et al. 2012] F. dell'Isola, P. Seppecher, and A. Madeo, "How contact interactions may depend on the shape of Cauchy cuts in $N$ th gradient continua: approach 'à la D'Alembert"', $Z$. Angew. Math. Phys. 63:6 (2012), 1119-1141.

[Forest 1998] S. Forest, "Mechanics of generalized continua: construction by homogenization", $J$. Phys. (France) IV 8:4 (1998), 39-48.

[Forest et al. 2011] S. Forest, N. M. Cordero, and E. P. Busso, "First vs. second gradient of strain theory for capillarity effects in an elastic fluid at small length scales", Comput. Mater. Sci. 50:4 (2011), 1299-1304. 
[Forte and Vianello 1996] S. Forte and M. Vianello, "Symmetry classes for elasticity tensors", J. Elasticity 43:2 (1996), 81-108.

[Forte and Vianello 1997] S. Forte and M. Vianello, "Symmetry classes and harmonic decomposition for photoelasticity tensors", Int. J. Eng. Sci. 35:14 (1997), 1317-1326.

[Geymonat and Weller 2002] G. Geymonat and T. Weller, "Classes de symétrie des solides piézoélectriques”, C. R. Acad. Sci. Paris Sér. I Math. 335:10 (2002), 847-852.

[Golubitsky et al. 1988] M. Golubitsky, I. Stewart, and D. G. Schaeffer, Singularities and groups in bifurcation theory, vol. 2, Applied Mathematical Sciences 69, Springer, New York, 1988.

[Gu and He 2011] S. T. Gu and Q. C. He, "Interfacial discontinuity relations for coupled multifield phenomena and their application to the modeling of thin interphases as imperfect interfaces", $J$. Mech. Phys. Solids 59:7 (2011), 1413-1426.

[Herman 1945] B. Herman, "Some theorems of the theory of anisotropic media", C. R. (Doklady) Acad. Sci. URSS (N. S.) 48 (1945), 89-92.

[Ihrig and Golubitsky 1984] E. Ihrig and M. Golubitsky, "Pattern selection with O(3) symmetry", Physica D 13:1-2 (1984), 1-33.

[Jerphagnon et al. 1978] J. Jerphagnon, D. Chemla, and R. Bonneville, "The description of the physical properties of condensed matter using irreducible tensors", Adv. Phys. 27:4 (1978), 609650 .

[Le Quang and He 2011] H. Le Quang and Q.-C. He, "The number and types of all possible rotational symmetries for flexoelectric tensors", Proc. R. Soc. Lond. A 467:2132 (2011), 2369-2386.

[Le Quang et al. 2012] H. Le Quang, N. Auffray, Q.-C. He, and G. Bonnet, "Symmetry groups and symmetry classes for sixth-order tensors", preprint, 2012. Submitted to Proc. R. Soc. Lond. A.

[Lebée and Sab 2011] A. Lebée and K. Sab, "A bending-gradient model for thick plates, I: Theory", Int. J. Solids Struct. 48 (2011), 2878-2888.

[Mindlin 1964] R. D. Mindlin, "Micro-structure in linear elasticity", Arch. Ration. Mech. Anal. 16 (1964), 51-78.

[Mindlin 1965] R. D. Mindlin, "Second gradient of strain and surface-tension in linear elasticity", Int. J. Solids Struct. 1:4 (1965), 417-438.

[Spencer 1970] A. J. M. Spencer, "A note on the decomposition of tensors into traceless symmetric tensors”, Int. J. Eng. Sci. 8 (1970), 475-481.

[Sternberg 1994] S. Sternberg, Group theory and physics, Cambridge University Press, Cambridge, 1994.

[Thionnet and Martin 2006] A. Thionnet and C. Martin, "A new constructive method using the theory of invariants to obtain material behavior laws", Int. J. Solids Struct. 43:2 (2006), 325-345.

[Toupin 1962] R. A. Toupin, "Elastic materials with couple-stresses", Arch. Ration. Mech. Anal. 11 (1962), 385-414.

[Triantafyllidis and Bardenhagen 1996] N. Triantafyllidis and S. Bardenhagen, "The influence of scale size on the stability of periodic solids and the role of associated higher order gradient continuum models", J. Mech. Phys. Solids 44:11 (1996), 1891-1928.

[Zheng and Boehler 1994] Q.-S. Zheng and J.-P. Boehler, "The description, classification, and reality of material and physical symmetries", Acta Mech. 102:1-4 (1994), 73-89.

Received 16 May 2012. Revised 2 Oct 2012. Accepted 2 Dec 2012. 
MARC OLIVE: molive@cmi.univ-mrs.fr

Laboratoire d'Analyse, Topologie, Probabilités, Centre de Mathématiques et Informatique,

CNRS, UMR 7353, Aix-Marseille Université, Technopôle Château-Gombert, 39 rue F. Joliot-Curie, 13453 Marseille, France

NICOLAS AUFFRAY: Nicolas.Auffray@univ-mlv.fr

Laboratoire Modélisation et Simulation Multi Echelle, Université Paris-Est, CNRS, UMR 8208, 5 boulevard Descartes, 77454 Marne-la-Vallée, France 